Check for updates

Cite this: Phys. Chem. Chem. Phys., 2017, 19, 19573

Received 23rd February 2017, Accepted 14th March 2017

DOI: $10.1039 / c 7 c p 01215 b$

rsc.li/pccp

\title{
A study of the water molecule using frequency control over nuclear dynamics in resonant $\mathrm{X}$-ray scattering
}

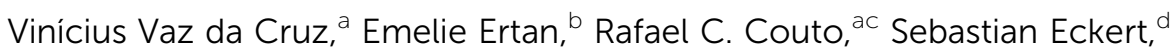 \\ Mattis Fondell, ${ }^{e}$ Marcus Dantz, ${ }^{f}$ Brian Kennedy, ${ }^{e}$ Thorsten Schmitt, ${ }^{f}$ \\ Annette Pietzsch, ${ }^{e}$ Freddy F. Guimarães, ${ }^{C}$ Hans Ågren, (D) ag Faris Gel'mukhanov, ${ }^{\text {ag }}$ \\ Michael Odelius, (D) *b Alexander Föhlisch*de and Victor Kimberg*a
}

\begin{abstract}
In this combined theoretical and experimental study we report a full analysis of the resonant inelastic X-ray scattering (RIXS) spectra of $\mathrm{H}_{2} \mathrm{O}, \mathrm{D}_{2} \mathrm{O}$ and $\mathrm{HDO}$. We demonstrate that electronically-elastic RIXS has an inherent capability to map the potential energy surface and to perform vibrational analysis of the electronic ground state in multimode systems. We show that the control and selection of vibrational excitation can be performed by tuning the X-ray frequency across core-excited molecular bands and that this is clearly reflected in the RIXS spectra. Using high level ab initio electronic structure and quantum nuclear wave packet calculations together with high resolution RIXS measurements, we discuss in detail the mode coupling, mode localization and anharmonicity in the studied systems.
\end{abstract}


vibrational modes coupling, ${ }^{37-39}$ resulting in a challenging analysis of the X-ray spectra. Due to this circumstance, a very important task of modern spectroscopy is to beat complexity by using the selectivity of X-ray excitation.

We have recently demonstrated ${ }^{40}$ that the three lowest intermediate core-excited states of gas-phase water can be considered as filters which single out excitations of a particular type of vibrational motion in the ground electronic state: bending, symmetric and antisymmetric stretching, something that can be applied for easier mapping of the PES of multimode molecular systems. In the present article, we go further and study the dynamical effect in RIXS by changing the excitation energy across particular resonances, which appears as an additional probe for the vibrational states in the ground electronic state and their localization in symmetric $\left(\mathrm{H}_{2} \mathrm{O}, \mathrm{D}_{2} \mathrm{O}\right)$ and asymmetric (HDO) systems. Here, we will show that, by means of the detuning control, one can reach vibrational levels up to the dissociation limit, which showcases the potential of using the RIXS technique to investigate the multi-dimensional potential surface of complex molecular systems.

In the present paper, we report a thorough and detailed analysis of vibrationally resolved electronically-elastic RIXS of gas-phase water, allowing for advanced studies of the molecular ground state. We do so by combining high-resolution experimental measurements over a broad range of excitation energies, high level $a b$ initio electronic structure methods and a multi-dimensional quantum wave packet description of the nuclear motion. Furthermore, in order to reach a more complete understanding of the nuclear dynamics upon core excitation of water, our study is extended by numerical simulations of the $\mathrm{D}_{2} \mathrm{O}$ and $\mathrm{HDO}$ isotopes. Indeed, as the electronic structures of the ground and core-excited states are the same for water and its isotope analogues, this provides an opportunity to study the RIXS spectral changes only in terms of dynamical features. In contrast to the symmetric $\mathrm{H}_{2} \mathrm{O}$ and $\mathrm{D}_{2} \mathrm{O}$ molecules, the symmetry breaking of HDO results in a localization of the stretching vibrations on the $\mathrm{OH}$ and $\mathrm{OD}$ bonds. One should note that although in HDO vibrational localization occurs in the ground state, vibrational delocalization is restored in certain core-excited states. ${ }^{41}$ Special attention is paid to the decay transitions from the $\mathrm{HO}$ and DO fragments of dissociation in the core-excited state. The corresponding RIXS feature known as the "atomic" peak ${ }^{42,43}$ is different in $\mathrm{H}_{2} \mathrm{O}, \mathrm{D}_{2} \mathrm{O}$ and HDO. The reason for this is the difference in the vibrational structure of these fragments as well as the slower dissociation of hydrogen in comparison with deuterium.

The paper is organized as follows. The details of the experiment are discussed in Section II. Modeling of electronic states and of nuclear dynamics in simulations of RIXS of gas-phase water is presented in Section III. Section IV collects the results of numerical simulations, their analysis including the comparison with the experiment and the results of simulations of isotopic substitutions of $\mathrm{H}_{2} \mathrm{O}$. Our findings are summarized in Section V.

\section{Experiment}

The experimental spectra presented here were measured using the SAXES spectrometer ${ }^{44}$ at the RIXS end station of the
ADRESS beam line ${ }^{45}$ at the Swiss Light Source. As described in our previous report, ${ }^{40}$ the $\mathrm{H}_{2} \mathrm{O}(\mathrm{g})$ sample was prepared by evacuation and heating of a $10 \mathrm{ml} \mathrm{H}_{2} \mathrm{O}(\mathrm{l})$ sample reservoir at $60^{\circ}$. The obtained gas was then transferred through previously evacuated and heated steel capillaries towards the interaction point. The sample volume was separated from the experimental chamber by a $150 \mathrm{~nm}$ thick silicon nitride membrane. We established a continuous sample replacement by constant evacuation of the $\mathrm{H}_{2} \mathrm{O}(\mathrm{l})$ sample reservoir, in turn generating a flow of fresh sample at the interaction volume. The signal emitted from the sample volume was detected using a spherical variable line space high-resolution RIXS spectrometer. The photon energy $\omega$ used for the excitation of the sample was scanned around the three lowest core-excited states $\left|1 \mathrm{a}_{1}{ }^{-1} 4 \mathrm{a}_{1}{ }^{1}\right\rangle,\left|1 \mathrm{a}_{1}{ }^{-1} 2 \mathrm{~b}_{2}{ }^{1}\right\rangle$, $\left|1 a_{1}{ }^{-1} 2 b_{1}{ }^{1}\right\rangle$ (also referred in what follows as $4 a_{1}, 2 b_{2}$ and $2 b_{1}$, respectively), extending over a total energy interval $533.25 \leq$ $\omega \leq 537.56 \mathrm{eV}$. The resonantly scattered photons were detected at a $90^{\circ}$ angle from the incoming photons with a combined experimental resolution of $75 \mathrm{meV}$.

\section{Theory}

The water molecule has three vibrational normal modes: bending (b), symmetric (s) and antisymmetric (a) stretching. It is known ${ }^{46,47}$ that the two stretching modes are strongly coupled (Darling-Dennison coupling) due to the close vibrational frequencies $\left(\omega_{\mathrm{s}}=3656.65 \mathrm{~cm}^{-1}\right.$ and $\left.\omega_{\mathrm{a}}=3755.79 \mathrm{~cm}^{-1}\right)$ and anharmonicity. The weaker coupling between the bending mode and the stretching motion allows one to treat the one-dimensional (1D) bending and the two-dimensional (2D) stretching motions separately in what we call a $2 \mathrm{D}+1 \mathrm{D}$ model. ${ }^{40} \mathrm{We}$ assume that the Born-Oppenheimer approximation is valid. Therefore, the electronic structure is described through the calculated $a b$ initio PESs (see Fig. 1 and the detailed description given in Section IIIA), while the nuclear degrees of freedom are treated by numerical time-dependent wave packet propagation ${ }^{48,49}$ (described in Section IIIC).

In the $2 \mathrm{D}+1 \mathrm{D}$ model, the $2 \mathrm{D}$ stretching motion is modeled by means of wave packet propagation on a two-dimensional PES, whereas simultaneously the bending mode is treated by solving the time-independent Schrödinger equation followed by calculations of the 1D Franck-Condon (FC) amplitudes. The $2 \mathrm{D}$ stretching dynamics is obtained by numerical solution of the time-dependent Schrödinger equation, ${ }^{50,51}$

$$
\imath \frac{\partial}{\partial t} \psi_{\mathrm{i}}(t)=h_{\mathrm{i}} \psi_{\mathrm{i}}(t)
$$

where $\mathrm{i}=\mathrm{c}$, $\mathrm{f}$ describes the core-excited and final states, respectively. The nuclear Hamiltonian is expressed in valence coordinates $^{52}$

$$
h_{\mathrm{i}}=-\frac{1}{2 \mu_{1}} \frac{\partial^{2}}{\partial R_{1}^{2}}-\frac{1}{2 \mu_{2}} \frac{\partial^{2}}{\partial R_{2}^{2}}-\frac{\cos \theta_{0}}{m_{\mathrm{O}}} \frac{\partial^{2}}{\partial R_{1} \partial R_{2}}+V_{\mathrm{i}}\left(R_{1}, R_{2}, \theta_{0}\right) \text {, }
$$

where $\theta_{0}=104.2^{\circ}$ is the ground state equilibrium bond angle, $R_{1}$ and $R_{2}$ are the bond distances $\left(R_{1}=\mathrm{OX}_{1}, R_{2}=\mathrm{OX}_{2}\right.$, with $\mathrm{X}=\mathrm{H}$ or $\left.\mathrm{D}\right)$, 

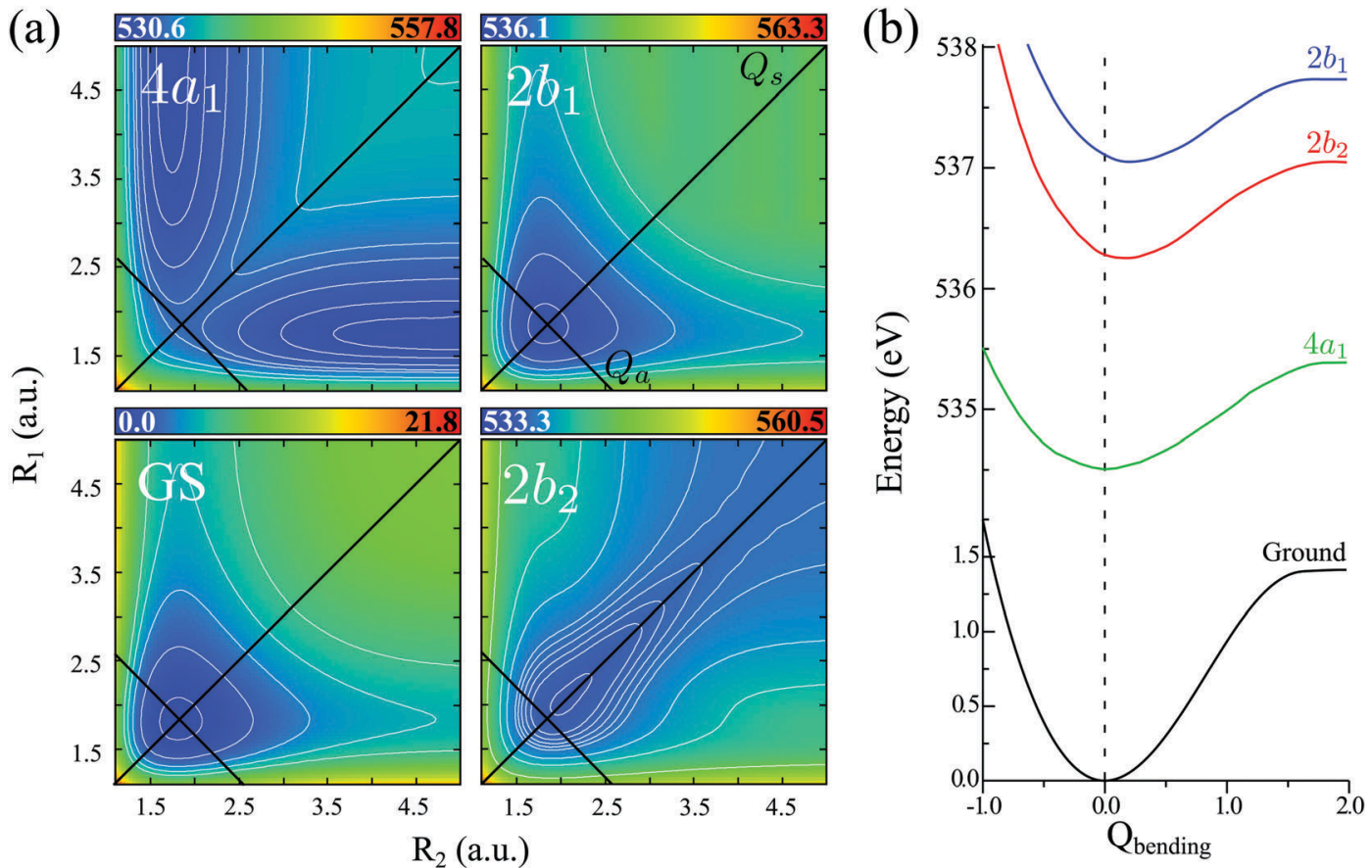

Fig. 1 Potentials of the ground and core-excited $\left(4 a_{1}, 2 b_{2}\right.$ and $\left.2 b_{1}\right)$ states of gas-phase water. (a) Potential energy surfaces representing the two stretching vibrational modes in valence coordinates $\left(R_{1}, R_{2}\right)$. The lines at the PESs show the direction of symmetric $\left(Q_{\mathrm{s}} \sim R_{1}+R_{2}\right)$ and antisymmetric $\left(Q_{\mathrm{a}} \sim R_{2}-R_{1}\right)$ vibrational normal modes, and the colored boxes above the PES show the energy range in eV, relative to the ground state minimum. (b) Potential energy curves of the bending mode.

and $V_{\mathrm{i}}\left(R_{1}, R_{2}, \theta_{0}\right)$ is the $2 \mathrm{D}$ PES of the $i$ th electronic state. The nuclear dynamics is sensitive to the reduced masses, which are defined as

$$
\mu_{1}=\frac{m_{1} m_{\mathrm{O}}}{m_{1}+m_{\mathrm{O}}}, \quad \mu_{2}=\frac{m_{2} m_{\mathrm{O}}}{m_{2}+m_{\mathrm{O}}}
$$

where $m_{1}=m_{2}=m_{\mathrm{H}}$ and $m_{1}=m_{2}=m_{\mathrm{D}}$ for $\mathrm{H}_{2} \mathrm{O}$ and $\mathrm{D}_{2} \mathrm{O}$, respectively, $m_{1}=m_{\mathrm{H}}$ and $m_{2}=m_{\mathrm{D}}$ for HDO. In contrast, the bending motion is treated by solving the time-independent $1 \mathrm{D}$ Schrödinger equation, along the bending coordinate, and computing the FC amplitudes $\left\langle\nu_{\mathrm{i}} \mid \nu_{\mathrm{i}^{\prime}}\right\rangle$ between the vibrational sub-levels of the electronic states $\mathrm{i}$ and $\mathrm{i}^{\prime}$. The PESs of the ground and coreexcited states are the same for the $\mathrm{H}_{2} \mathrm{O}, \mathrm{D}_{2} \mathrm{O}$ and $\mathrm{HDO}$ molecules (they could potentially differ only due to a weak breakdown of the Born-Oppenheimer approximation).

\section{A. Electronic structure and potential energy surfaces}

The electronic structure calculations presented in this article were performed using RASPT2 calculations, in which static electron correlation is treated variationally within the restricted-activespace self-consistent field (RASSCF) framework ${ }^{53,54}$ and dynamical electron correlation is included in a perturbative fashion. The RASSCF orbital space is subdivided into three smaller sections with different restrictions applied to the MOs in these subspaces. The three subspaces are referred to as RAS1, where the number of allowed (electrons) holes is restricted, RAS2, where a full CI is performed (all possible permutations of electrons are allowed) and RAS3, where a restricted number of electrons is admitted.
Hence, the choice of the orbital space and the partitioning of the active orbitals are of great importance in these calculations.

In the water molecule, in order to study the states we were interested in, at the very least, the consideration of 8 MOs O1s $\left(1 a_{1}\right), 2 a_{1}, 3 a_{1}, 1 b_{2}, 1 b_{1}, 4 a_{1}, 2 b_{2}, 2 b_{1}$, however, to improve the description of the states, additional higher energy MOs were also included. In particular, the ground state geometry optimization and the determination of the normal vibrational modes were performed with an active space of 8 electrons in 9 active orbitals, with the $1 \mathrm{a}_{1}$ MO placed in the inactive space. In the calculation of the PESs, we included three additional MOs in the active space, resulting in an active space of 10 electrons in 11 active orbitals. The $1 \mathrm{a}_{1}$ orbital, which was frozen from the Hartree-Fock calculation, was placed in RAS3 and the ground state and the three core-excited states were generated in two separate RASSCF calculations with two and one electrons occupying RAS3, respectively. RASSCF state-averaging was employed over the $\left|1 \mathrm{a}_{1}{ }^{-1} 4 \mathrm{a}_{1}{ }^{1}\right\rangle$ and $\left|1 \mathrm{a}_{1}{ }^{-1} 2 \mathrm{~b}_{2}{ }^{1}\right\rangle$ core-excited states. All calculations were performed employing $C_{\mathrm{s}}$ symmetry and Cholesky decomposition of the two-electron integrals.

To accurately represent the dynamic electron correlation, we employed the CAS/RAS-PT2 scheme ${ }^{55,56}$ for all electronic structure calculations, wherein second-order multi-configurational perturbation is performed on the reference states (CAS/RASSCF). To avoid the problems introduced by intruder states (weakly interacting), an imaginary shift of 0.1 a.u. was used. Multi-state RASPT2 was performed over the $\left|1 \mathrm{a}_{1}{ }^{-1} 4 \mathrm{a}_{1}{ }^{1}\right\rangle$ and $\left|1 \mathrm{a}_{1}{ }^{-1} 2 \mathrm{~b}_{2}{ }^{1}\right\rangle$ core-excited states. Spin-free energy eigenstates and the transition dipole moments were calculated in the RAS state interaction program (RASSI). ${ }^{57,58}$ 
To obtain correct excitation energies for the core-excited states, relativistic effects should be accounted for. Scalar relativistic effects were included by using the ANO-RCC ${ }^{59}$ relativistic basis set in combination with the Douglas-Kroll-Hess approach, ${ }^{60,61}$ wherein block-diagonalization of the relativistic (Dirac) Hamiltonian by a unitary operator transforms the fourcomponent relativistic wave function to a two-component form and mean-field integrals were computed using the atomic mean field (AMFI) ${ }^{62}$ approximation.

The presence of low-lying electronically excited states of the Rydberg character in the water molecule ${ }^{63}$ made it necessary to employ a basis set that could describe the character of both the valence excited states and the lowest Rydberg excited states. To this end, a (2s2p1d) Rydberg basis set was generated according to the steps described in ref. 64, and used in combination with the main ANO-RCC basis set for the calculation of the PESs. All calculations described in this section were performed using the MOLCAS 8.0 software. $^{65}$

\section{B. Spectrum of the ground state stretching modes}

The vibrational spectrum related to the $2 \mathrm{D}$ stretching potential (Fig. 1) of the ground state $\mathrm{H}_{2} \mathrm{O}$ is shown in Fig. 2a and summarized in more detail in Table 1, where it is compared with previous studies. ${ }^{66}$ For the very lowest vibrational levels of the ground electronic state, the harmonic approximation is applicable, and as a result the $2 \mathrm{D}$ stretching motion can be modeled as two uncoupled harmonic oscillators - symmetric and antisymmetric - described by the normal coordinates $Q_{\mathrm{s}} \propto R_{1}+R_{2}$ and $Q_{\mathrm{a}} \propto R_{2}-R_{1}$, respectively. However, this model fails to explain the spectrum starting from the $n=3$ vibrational group due to the anharmonicity of the PES (see the PES shape in Fig. 1). In spite of this, it is useful to keep the assignment of the $2 \mathrm{D}$ vibrational states $\psi_{n_{\mathrm{s}}, n_{\mathrm{a}}}$ based on the quantum numbers $n_{\mathrm{s}}$ and $n_{\mathrm{a}}$ of the symmetric and antisymmetric stretching modes. ${ }^{67}$ Fig. 2 a shows that the stretching vibrational energy levels of the ground state are grouped according to the group number $n$

$$
n=n_{\mathrm{s}}+n_{\mathrm{a}} .
$$

Each $n$th group consists of $n+1$ vibrational levels characterized by two quantum numbers $\left(n_{\mathrm{s}}, n_{\mathrm{a}}\right)=\left(n-n_{\mathrm{a}}, n_{\mathrm{a}}\right)$. For example, at $n=3$ we have four energy levels assigned as $(3,0),(2,1),(1,2)$ and $(0,3)$.

The energy spacing between the vibrational states has a peculiar behavior. A strict solution of the eigenvalue problem for the $2 \mathrm{D}$ stretching potential of $\mathrm{H}_{2} \mathrm{O}$ gives a qualitatively different spectrum (Fig. 2a) compared to the one predicted by the independent stretching mode approximation, which gives a monotonically varying energy spacing. Within the $n$th group $(n \geq 3)$, a degeneracy of the low lying energy vibrational states $\psi_{n, 0}$ and $\psi_{n-1,1}$ can be observed (Table 1 ). This degeneracy is accompanied by bifurcations of the ground state vibrational (a)

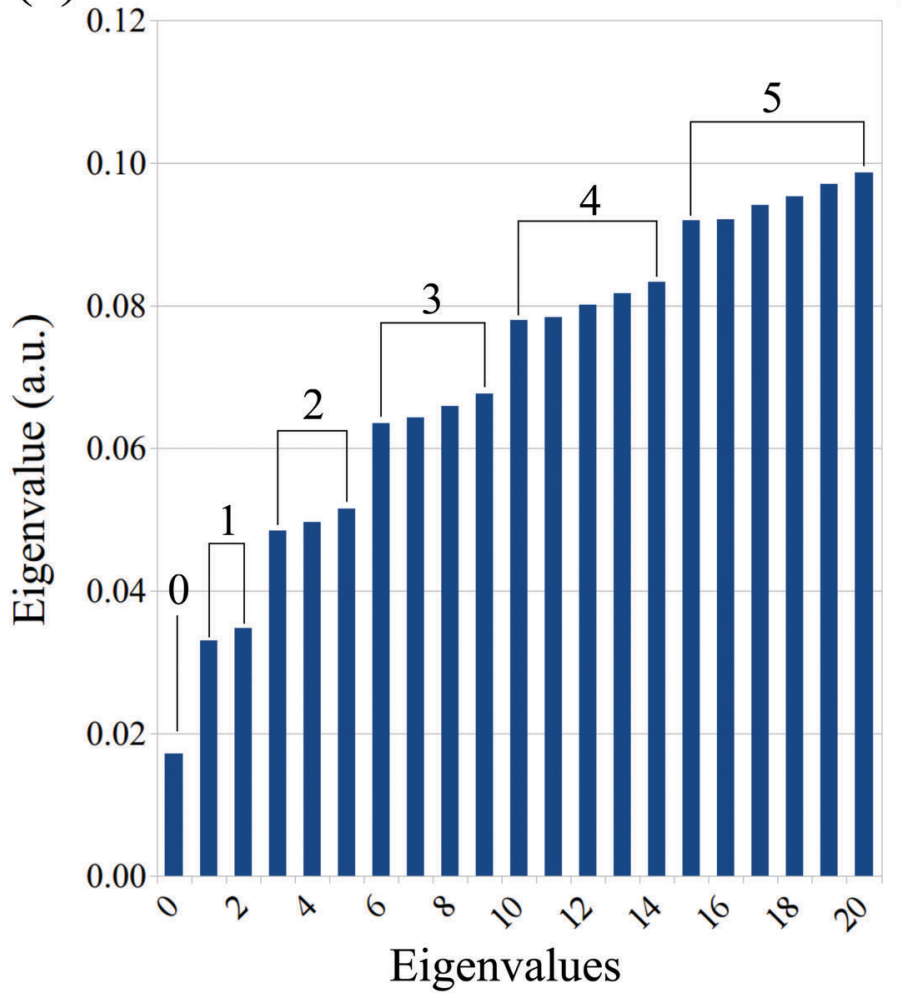

(b)
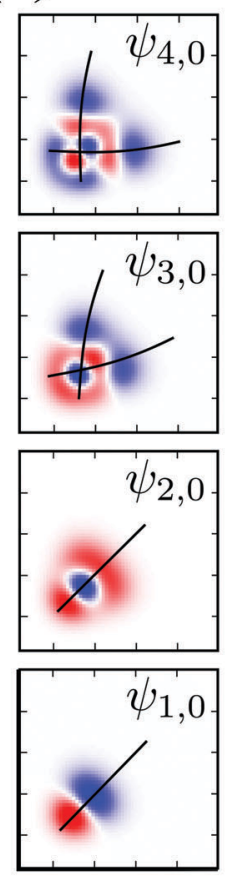

(c)
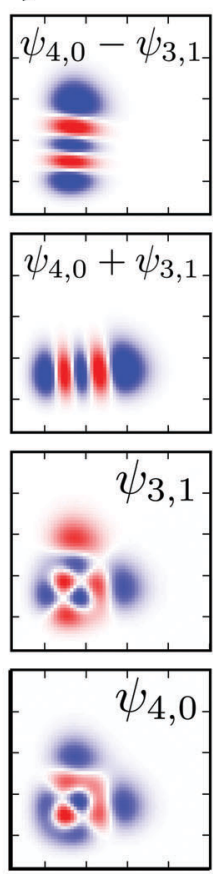

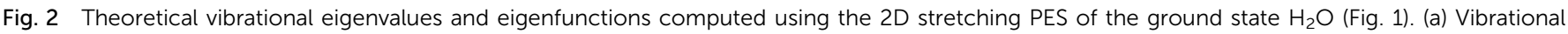

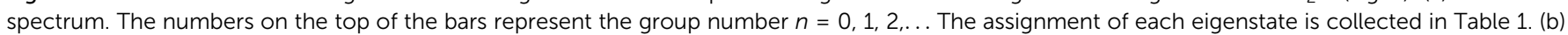
Bifurcation of the vibrational wave function, illustrated by the black lines. (c) Vibrational mode localization: delocalized vs localized representation. 
Table 1 Computed vibrational energies of the 2D stretching potential of the ground state of $\mathrm{H}_{2} \mathrm{O}$ (Fig. 1), in comparison with the theoretical (Theo.) and experimental (Exp.) values taken from ref. 66. $N$ is the number of eigenstates and $n$ is the group number (see Fig. 2a). The vibrational energies (in $\mathrm{eV}$ ) are given with respect to the zero-point energy

\begin{tabular}{|c|c|c|c|c|c|}
\hline$n$ & $N$ & $\left(n_{\mathrm{s}}, n_{\mathrm{a}}\right)$ & This work & Theo. ${ }^{66}$ & Exp. ${ }^{66}$ \\
\hline \multirow[t]{2}{*}{1} & 1 & $(1,0)$ & 0.4588 & 0.4532 & 0.4531 \\
\hline & 2 & $(0,1)$ & 0.4680 & 0.4654 & 0.4654 \\
\hline \multirow[t]{3}{*}{2} & 3 & $(2,0)$ & 0.9003 & 0.8924 & 0.8923 \\
\hline & 4 & $(1,1)$ & 0.9037 & 0.8983 & 0.8983 \\
\hline & 5 & $(0,2)$ & 0.9295 & 0.9224 & 0.9224 \\
\hline \multirow[t]{4}{*}{3} & 6 & $(3,0)$ & 1.3222 & 1.3134 & 1.3133 \\
\hline & 7 & $(2,1)$ & 1.3229 & 1.3151 & 1.3150 \\
\hline & 8 & $(1,2)$ & 1.3581 & 1.3467 & 1.3467 \\
\hline & 9 & $(0,3)$ & 1.3763 & 1.3669 & 1.3669 \\
\hline \multirow[t]{5}{*}{4} & 10 & $(4,0)$ & 1.7241 & 1.7133 & 1.7133 \\
\hline & 11 & $(3,1)$ & 1.7242 & 1.7136 & 1.7137 \\
\hline & 12 & $(2,2)$ & 1.7769 & 1.7621 & 1.7620 \\
\hline & 13 & $(1,3)$ & 1.7869 & 1.7741 & 1.7741 \\
\hline & 14 & $(0,4)$ & 1.8141 & 1.8011 & 1.8012 \\
\hline \multirow[t]{6}{*}{5} & 15 & $(5,0)$ & 2.1070 & 2.0936 & 2.0937 \\
\hline & 16 & $(4,1)$ & 2.1070 & 2.0936 & 2.0938 \\
\hline & 17 & $(3,2)$ & 2.1807 & 2.1631 & 2.1631 \\
\hline & 18 & $(2,3)$ & 2.1841 & 2.1676 & 2.1677 \\
\hline & 19 & $(1,4)$ & 2.2153 & 2.1990 & 2.1990 \\
\hline & 20 & $(0,5)$ & 2.2408 & 2.2237 & - \\
\hline \multirow[t]{7}{*}{6} & 21 & $(6,0)$ & 2.4702 & 2.4507 & 2.4509 \\
\hline & 22 & $(5,1)$ & 2.4702 & 2.4507 & 2.4509 \\
\hline & 23 & $(4,2)$ & 2.5647 & 2.5440 & 2.5442 \\
\hline & 24 & $(3,3)$ & 2.5654 & 2.5418 & 2.5453 \\
\hline & 25 & $(2,4)$ & 2.6102 & 2.5903 & - \\
\hline & 26 & $(1,5)$ & 2.6271 & 2.6069 & - \\
\hline & 27 & $(0,6)$ & 2.6579 & 2.6357 & - \\
\hline
\end{tabular}

wave functions $\psi_{n, 0}$ and $\psi_{n-1,1}$, where one can find density lobes localized along the bonds $R_{1}$ and $R_{2}$ (Fig. 2b). This phenomenon is known as the normal-to-local mode transition. ${ }^{67,68}$ The degeneracy of the states means that the delocalized $\psi_{n, 0}, \psi_{n-1,1}$ and localized

$$
\psi_{R_{1}}=\frac{\psi_{n, 0}-\psi_{n-1,1}}{\sqrt{2}}, \quad \psi_{R_{2}}=\frac{\psi_{n, 0}+\psi_{n-1,1}}{\sqrt{2}}
$$

vibrational states can be equally used (Fig. 2c). Apparently, the real localization of the vibrations along the bonds $R_{1}$ and $R_{2}$ happens in the case of perturbations comparable with the small spacing between the delocalized states. For example, this happens in liquid water and for the HDO molecule (see Section IVD). As it will be shown (Section IVB), the bifurcation of vibrational states (Fig. 2b and c) is also important for the understanding of the propensity rules in the RIXS spectra.

\section{RIXS cross section}

In the $2 \mathrm{D}+1 \mathrm{D}$ model, the total vibrational wave function is the product of the bending wave function $\left|\nu_{\mathrm{i}}\right\rangle$ and the stretching one $\left|\psi_{n_{s}, n_{\mathrm{a}}}\right\rangle$. The initial state of the presently investigated electronically-elastic RIXS process is the lowest vibrational level $|0\rangle\left|\psi_{0,0}\right\rangle$ of the ground electronic state, while the final RIXS state is the vibrationally excited state

$$
\left|\nu_{\mathrm{f}}\right\rangle\left|\psi_{n_{\mathrm{s}}, n_{\mathrm{a}}}\right\rangle .
$$

This factorization is also valid for the core-excited state.

The RIXS cross section is given by the Kramers-Heisenberg equation, ${ }^{18}$ which can be written in the $2 \mathrm{D}+1 \mathrm{D}$ model as

$$
\begin{aligned}
\sigma\left(\omega^{\prime}, \omega\right) & =\sum_{\nu_{\mathrm{f}} ; n_{\mathrm{s}}, n_{\mathrm{a}}}\left|F_{\nu_{\mathrm{f}} ; n_{\mathrm{s}}, n_{\mathrm{a}}}\right|^{2} \Delta\left(\omega-\omega^{\prime}-\varepsilon_{\nu_{\mathrm{f}}}-\varepsilon_{n_{\mathrm{s}} n_{\mathrm{a}}}+\varepsilon_{0}, \Gamma_{\mathrm{f}}\right), \\
F_{\nu_{\mathrm{f}} ; n_{\mathrm{s}}, n_{\mathrm{a}}} & =-l \sum_{\nu_{\mathrm{c}}}\left\langle\nu_{\mathrm{f}} \mid \nu_{\mathrm{c}}\right\rangle\left\langle\nu_{\mathrm{c}} \mid 0\right\rangle\left\langle\psi_{n_{\mathrm{s}} n_{\mathrm{a}}} \mid \Psi_{\nu_{\mathrm{c}}}(0)\right\rangle .
\end{aligned}
$$

Here, $\Delta(\Omega, \Gamma)=\Gamma /\left(\pi\left(\Omega^{2}+\Gamma^{2}\right)\right), \omega$ and $\omega^{\prime}$ are the frequencies of incoming and scattered photons, respectively, $\varepsilon_{0}$ is the total zeropoint energy of the ground state, $\varepsilon_{\nu_{\mathrm{f}}}$ and $\varepsilon_{n_{\mathrm{s}}} n_{\mathrm{a}}$ are the vibrational energies of the bending and stretching final states, respectively, and $\Gamma_{\mathrm{f}}$ is the lifetime broadening of the final state. In this paper, we are concerned with the vibrational profile of the RIXS process, thus in the above equation we omitted a prefactor proportional to the transition dipole moment and describing experimental geometry, which is the same for each vibrational resonance in the Born-Oppenheimer approximation. The RIXS amplitude $F_{\nu_{\tilde{f}} n_{\mathrm{s}}, n_{\mathrm{a}}}$ is defined by the FC amplitudes $\left\langle\nu_{\mathrm{f}} \mid \nu_{\mathrm{c}}\right\rangle\left\langle\nu_{\mathrm{c}} \mid 0\right\rangle$ for the bending modes and the overlap of the $2 \mathrm{D}$ time-integrated wave packet

$$
\left|\Psi_{\nu_{\mathrm{c}}}(0)\right\rangle=\int_{0}^{\infty} \mathrm{e}^{l\left(\omega-\omega_{\mathrm{c} 0}+\varepsilon_{0}-\varepsilon_{\nu_{\mathrm{c}}}+i \Gamma\right) t}\left|\psi_{\mathrm{c}}(t)\right\rangle \mathrm{d} t
$$

with the 2D stretching vibrational state $\psi_{n_{s} n_{\mathrm{a}}}$ of the ground electronic state. The wave packet $\left|\Psi_{\nu_{c}}(0)\right\rangle$ depends on the dynamics of the core-excited wave packet $\left|\psi_{\mathrm{c}}(t)\right\rangle=\exp \left(-l h_{\mathrm{c}} t\right)|0\rangle$ and on the incoming photon frequency $\omega$. In eqn (8), $\omega_{\mathrm{c} 0}$ is the frequency of the transition between the minima of the ground and core-excited state potentials. In the simulations, we consider the core-hole lifetime broadening $\Gamma=0.079 \mathrm{eV}$ (HWHM) ${ }^{69}$ Using the time-dependent representation for the Lorentzian $\Delta(\Omega, \Gamma)=\pi^{-1} \operatorname{Re} \int_{0}^{\infty} \exp (\imath(\Omega+\imath \Gamma) t) \mathrm{d} t$, the RIXS cross section can be written as ${ }^{48,49}$

$$
\begin{aligned}
\sigma\left(\omega^{\prime}, \omega\right)= & \frac{1}{\pi} \sum_{\nu_{\mathrm{f}}, \nu_{\mathrm{c}}^{\prime}, \nu_{\mathrm{c}}}\left\langle 0 \mid \nu_{\mathrm{c}}^{\prime}\right\rangle\left\langle\nu_{\mathrm{c}}^{\prime} \mid \nu_{\mathrm{f}}\right\rangle\left\langle\nu_{\mathrm{f}} \mid \nu_{\mathrm{c}}\right\rangle\left\langle\nu_{\mathrm{c}} \mid 0\right\rangle \\
& \times \operatorname{Re} \int_{0}^{\infty} \mathrm{e}^{l\left(\omega-\omega^{\prime}-\varepsilon_{\nu_{\mathrm{f}}}+\varepsilon_{0}+I \Gamma_{\mathrm{f}}\right) t} \sigma_{\nu_{\nu_{\mathrm{c}}} \nu_{\mathrm{c}}}(t) \mathrm{d} t
\end{aligned}
$$

with the autocorrelation function

$$
\sigma_{\nu_{\mathrm{c}}^{\prime} \nu_{\mathrm{c}}}(t)=\left\langle\Psi_{\nu_{\mathrm{c}}^{\prime}}(0)\left|\mathrm{e}^{-\imath h_{\mathrm{f}} t}\right| \Psi_{\nu_{\mathrm{c}}}(0)\right\rangle .
$$

\section{Symmetry selection rules and propensity rules}

The PESs of $\mathrm{H}_{2} \mathrm{O}$ (Fig. 1) is symmetric with respect to the reflection $Q_{\mathrm{a}} \rightarrow-Q_{\mathrm{a}}$ relative to the $Q_{\mathrm{s}}$ axis. This results in the following symmetry property of the stretching vibrational wave function of the ground state ${ }^{40}$

$$
\psi_{n_{\mathrm{s}} n_{\mathrm{a}}}\left(Q_{\mathrm{s}},-Q_{\mathrm{a}}\right)=(-1)^{n_{a}} \psi_{n_{\mathrm{s}} n_{\mathrm{a}}}\left(Q_{\mathrm{s}}, Q_{\mathrm{a}}\right) .
$$

Due to this symmetry, only RIXS transitions to states with even $n_{\mathrm{a}}$ are allowed $\left(n_{\mathrm{a}}=0,2,4 \ldots\right)$ as the process starts from the lowest vibrational state $\psi_{0,0}$. 
On top of the strict symmetry (parity) selection rules additional propensity rules can be applied in the case of RIXS, depending on the relative shapes of the core-excited wavepacket (8) and the final anharmonic vibrational wave functions (Fig. 2). They will be discussed in detail by comparing the wave packet dynamics in the dissociative $4 \mathrm{a}_{1}$ and bound $2 \mathrm{~b}_{2}$ coreexcited states (Section IVB).

\section{E. Details of numerical simulations}

All wave packet propagations were computed employing the eSPec program, ${ }^{51,70}$ which uses a second-order propagation scheme and a discrete variable representation (DVR) of the wave functions. ${ }^{70,71}$ The kinetic energy operator in (2) was implemented considering fourth-order $\mathcal{O}\left(\Delta R^{4}\right)$ finite difference numerical derivatives. For the one-dimensional cases, direct diagonalization of the Hamiltonian was carried out to obtain the vibrational eigenstates, for the $2 \mathrm{D}$ potentials the Lanczos tridiagonalization algorithm was used. ${ }^{71}$ The wave packets were discretized with coordinate steps of $\Delta R_{1}=$ $\Delta R_{2}=4.0 \times 10^{-2}$ a.u. on a $320 \times 320$ grid and were propagated with a time step of $\Delta t=5.0 \times 10^{-4}$ fs. Absorbing boundary conditions $^{72}$ were used for the dissociative potential in order to avoid non-physical reflections at the edges of the spatial grid.

Because both $\omega$ and $\omega^{\prime}$ lie close to the X-ray absorption profile, the absorption of incoming and scattered photons is important. We took into account the self-absorption for the scattering cross section using the equation ${ }^{73-75}$

$$
\tilde{\sigma}\left(\omega^{\prime}, \omega\right)=\frac{\sigma\left(\omega^{\prime}, \omega\right)}{1+\frac{\sigma_{\mathrm{abs}}\left(\omega^{\prime}\right)}{\sigma_{\mathrm{abs}}(\omega)}}
$$

where the RIXS cross section $\sigma\left(\omega^{\prime}, \omega\right)$ is computed using eqn (9). Similar to the RIXS, the X-ray absorption spectrum (XAS) $\sigma_{\text {abs }}(\omega)$ was simulated using the time-dependent wave packet technique with the autocorrelation function $\sigma_{\nu_{\mathrm{c}}}(t)=\left\langle\psi_{0,0} \mid \psi_{\mathrm{c}}(t)\right\rangle$

$$
\sigma_{\mathrm{abs}}(\omega)=d_{\mathrm{c} 0}^{2} \sum_{\nu_{\mathrm{c}}}\left|\left\langle\nu_{\mathrm{c}} \mid 0\right\rangle\right|^{2} \operatorname{Re} \int_{0}^{\infty} \mathrm{e}^{l\left(\omega-\omega_{\mathrm{c} 0}+\varepsilon_{0}-\varepsilon_{\nu_{\mathrm{c}}}+I \Gamma\right) t} \sigma_{\nu_{\mathrm{c}}}(t) \mathrm{d} t,
$$

where $d_{\mathrm{co}}$ is the transition dipole moment.

In order to reproduce the experimental profile, the theoretical RIXS cross section (12) was convoluted using the instrumental function $\Phi\left(\omega_{1}{ }^{\prime}-\omega^{\prime}\right)$, which we approximated by using the Gaussian function:

$$
\begin{gathered}
\sigma^{\prime}\left(\omega^{\prime}, \omega\right)=\int \tilde{\sigma}\left(\omega_{1}^{\prime}, \omega\right) \Phi\left(\omega_{1}^{\prime}-\omega^{\prime}\right) \mathrm{d} \omega_{1}^{\prime}, \\
\Phi\left(\omega_{1}^{\prime}-\omega^{\prime}\right)=\frac{2}{\Delta} \sqrt{\frac{\ln 2}{\pi}} \exp \left(-\frac{\left(\omega_{1}^{\prime}-\omega^{\prime}\right)^{2} 4 \ln 2}{\Delta^{2}}\right)
\end{gathered}
$$

with the total experimental broadening $\Delta=0.098 \mathrm{eV}$ taken as the FWHM of the $0-0$ elastic peak $\left(\omega-\omega^{\prime}=0\right)$.

\section{Results}

The RIXS spectrum is very sensitive to nuclear dynamics in the intermediate core-excited state, and due to this we start with an analysis of the vibrationally resolved XAS (13). The computed near-edge X-ray absorption spectrum of the gas-phase $\mathrm{H}_{2} \mathrm{O}$ (Fig. 3a) consists of three bands corresponding to the three

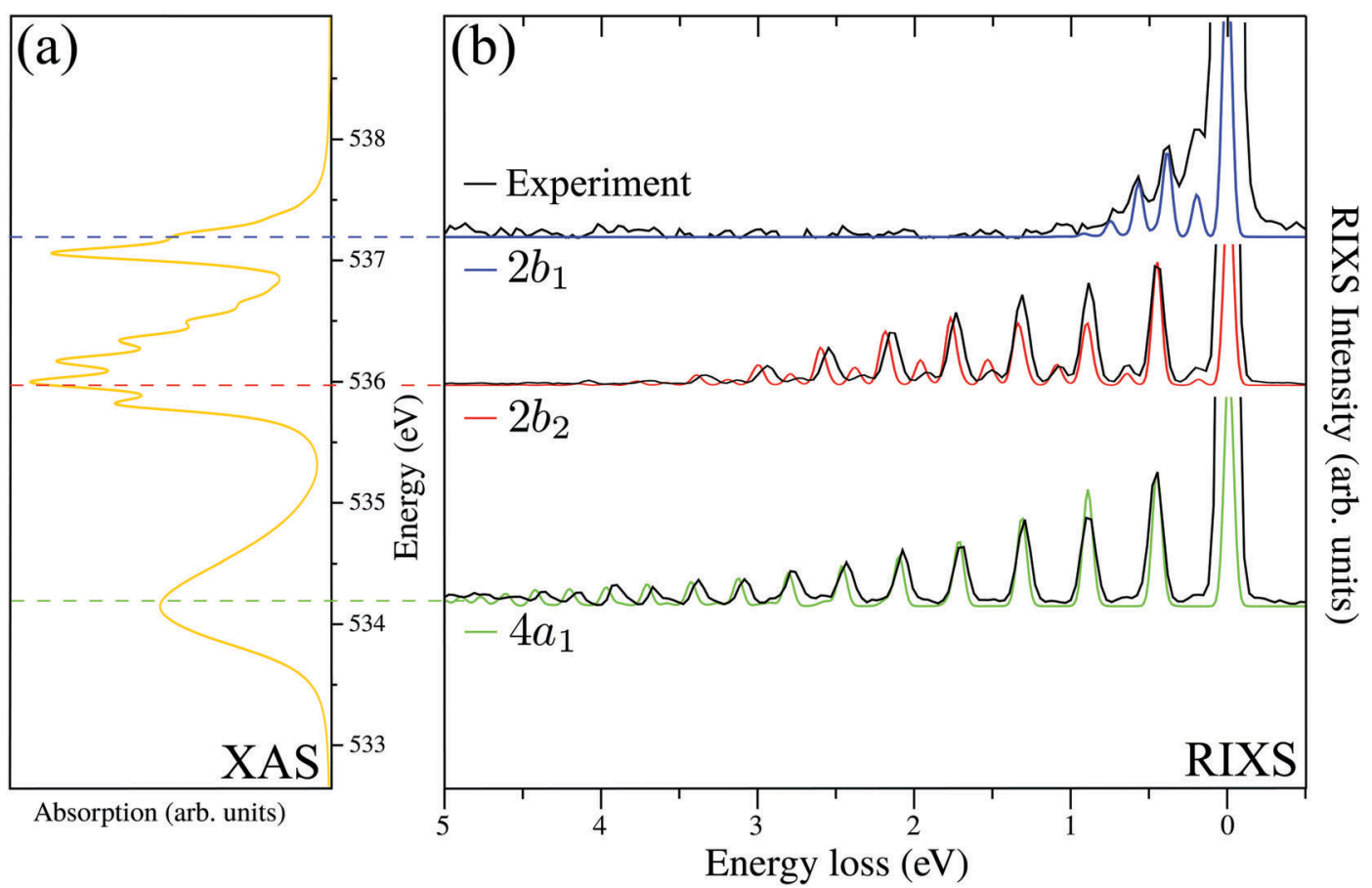

Fig. 3 (a) Theoretical near edge $X$-ray absorption spectrum of gas-phase $\mathrm{H}_{2} \mathrm{O}: 4 \mathrm{a}_{1}, 2 \mathrm{~b}_{2}$ and $2 \mathrm{~b}_{1}$ resonances. The dashed lines represent the excitation energy considered in the RIXS spectra presented in panel (b), for each one of the core-excited states. (b) Theoretical and experimental RIXS at $2 b_{1}$, $2 b_{2}$ and $4 \mathrm{a}_{1}$ resonances, for detuning $\Omega_{2 \mathrm{~b}_{1}}=+0.20 \mathrm{eV}, \Omega_{2 \mathrm{~b}_{2}}=-0.025 \mathrm{eV}$ and $\Omega_{4 \mathrm{a}_{1}}=+0.05 \mathrm{eV}$. Here and below, the energy loss is defined as $\omega-\omega^{\prime}$. 
lowest core-excited states $4 \mathrm{a}_{1}, 2 \mathrm{~b}_{2}$ and $2 \mathrm{~b}_{1}$, in agreement with previous experimental and theoretical studies. ${ }^{13,15}$ In order to reproduce the relative intensity of the three core-excited resonances, an appropriate ratio of the transition dipole moments has to be taken into account. In our simulations, we use the following ratio of the squared electronic transition dipole moments ${ }^{76} d_{4 \mathrm{a}_{1}, 0}{ }^{2}: d_{2 \mathrm{~b}_{2}, 0}{ }^{2}: d_{2 \mathrm{~b}_{1}, 0}{ }^{2}=1: 1.37: 0.57$.

Due to the dissociative character of the $4 \mathrm{a}_{1}$ core-excited state, this state is presented in the XAS as a broad band centered at $\omega=534.15 \mathrm{eV}$ without any vibrational structure. In contrast, the $2 b_{2}$ state has a bound character with the PES strongly different from the ground state potential (Fig. 1). Due to this, the XAS profile (maximum at $536.00 \mathrm{eV}$ ) displays a rather long vibrational progression. The narrow absorption peak at $537.06 \mathrm{eV}$ corresponds to the transition to the bound $2 b_{1}$ state. The reason for the suppression of the vibrational structure for this band is the nearly parallel PESs of the ground and $2 b_{1}$ core-excited states (Fig. 1). The nuclear dynamics in the core-excited state, and hence the RIXS spectral profile, also depends strongly on the detuning

$$
\Omega=\omega-\omega_{\mathrm{c}}^{\mathrm{XAS}}
$$

of the excitation energy $\omega$ with respect to the maximum of X-ray absorption band $\left(\omega_{\mathrm{c}}^{\mathrm{XAS}}\right)$ of each core-excited state, as it will become apparent in the following sections.

\section{A. RIXS of $\mathrm{H}_{2} \mathrm{O}$ near the top of the $2 b_{1}, 2 b_{2}$ and $4 a_{1}$ resonances}

Fig. $3 \mathrm{~b}$ collects the experimental and theoretical RIXS spectra near the top of the $2 \mathrm{~b}_{1}, 2 \mathrm{~b}_{2}$ and $4 \mathrm{a}_{1}$ resonances: $\Omega_{2 \mathrm{~b}_{1}}=+0.20 \mathrm{eV}$, $\Omega_{2 \mathrm{~b}_{2}}=-0.025 \mathrm{eV}$ and $\Omega_{4 \mathrm{a}_{1}}=+0.05 \mathrm{eV}$.

$2 b_{1}$ resonance. The RIXS spectrum at the $2 b_{1}$ resonance (Fig. 3b upper panel) shows a short progression of about $0.7 \mathrm{eV}$. This progression arises solely from the excitation of the bending vibrational mode. The reason for this is that the stretching PESs of the ground and $2 b_{1}$ core-excited states are nearly parallel. Therefore, the stretching modes are almost not excited at all in the course of the core excitation and, hence, mainly the $(0,0)-(0,0)-(0,0)$ stretching transition is seen in RIXS. This effect is seen clearly in the $2 \mathrm{D}$ stretching core-excited wave packet dynamics (Fig. 4): the $2 b_{1}$ wave packet barely moves, remaining around the minimum position of the ground state PES. This indicates that the RIXS at the $2 b_{1}$ resonance can be used to separately study the bending vibrational mode of water.

$\mathbf{2 b}_{\mathbf{2}}$ resonance. The RIXS spectrum (Fig. 3b mid panel) shows two vibrational progressions at the $2 b_{2}$ resonance. The one with the smaller frequency $(\sim 0.2 \mathrm{eV})$ corresponds to the excitation of the bending mode, and the higher intensity progression with a frequency of about $0.45 \mathrm{eV}$ corresponds to the stretching vibrations.

$4 a_{1}$ resonance. The RIXS spectrum at the $4 \mathrm{a}_{1}$ resonance (Fig. 3b lower panel) shows only stretching progression. A strong suppression of the bending modes is caused by the almost identical shapes of the bending mode potentials of the $4 \mathrm{a}_{1}$ core-excited and ground states (Fig. 1).

\section{B. Spatial distribution of the core-excited wave packet and propensity rule}

One can discern an interesting effect by comparing the RIXS spectra at the $2 b_{2}$ and $4 a_{1}$ resonances (see Fig. 5). Both experiment and theory display a shift between these spectra for $\omega-\omega^{\prime}>1 \mathrm{eV}(n>2)$. To understand this phenomenon, let us scrutinize the stretching states $\psi_{n_{\mathrm{s}}, n_{\mathrm{a}}}$ involved in the formation of the RIXS spectrum.

The bars in the zoomed RIXS spectra (Fig. 5b) show the positions and strengths of the stretching states which contribute to the observed peak. The strength of the resonance is given by the overlap $\left|\left\langle\psi_{n_{\mathrm{s}}, n_{\mathrm{a}}} \mid \Psi_{0}(0)\right\rangle\right|^{2}$ between the core-excited wave packet $\Psi_{0}(0)$ and the ground state wave functions $\psi_{n_{\mathrm{s}}, n_{\mathrm{a}}}$ (see eqn (7)). According to the selection rules (Section IIID), transitions to the states with odd $n_{\mathrm{a}}$ are forbidden in the RIXS via both $4 \mathrm{a}_{1}$ and $2 \mathrm{~b}_{2}$ states. In order to explain the observed spectral shift we have to address additional propensity rules arising from the qualitatively different shape of the core-excited wave packet in the $4 a_{1}$ and $2 b_{2}$ states. Indeed, the RIXS process at the $4 \mathrm{a}_{1}$ resonance populates mainly the states $\psi_{n_{\mathrm{s}}, 0}$, which are suppressed in the RIXS spectrum at the $2 b_{2}$ resonance, where the main contributions
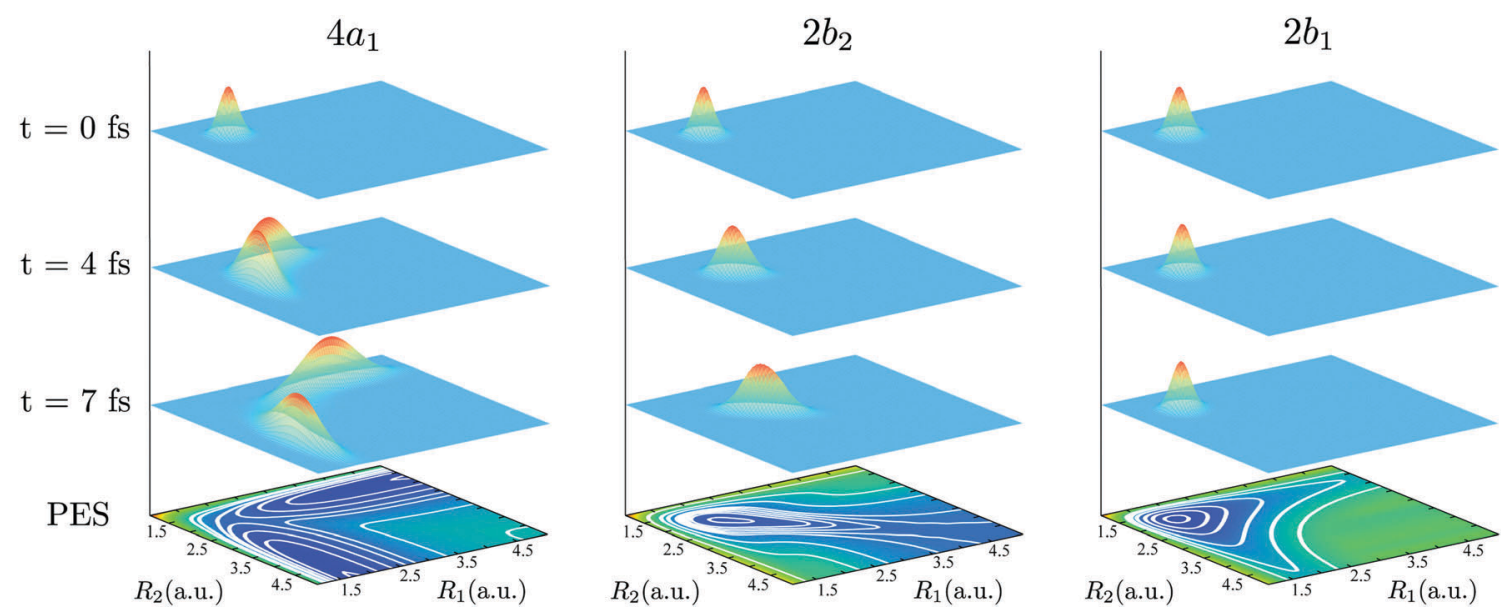

Fig. 4 Wave packet dynamics $\left(\left|\psi_{c}(t)\right|^{2}\right)$ in the core-excited states $4 a_{1}, 2 b_{2}$ and $2 b_{1}$. 
(a)

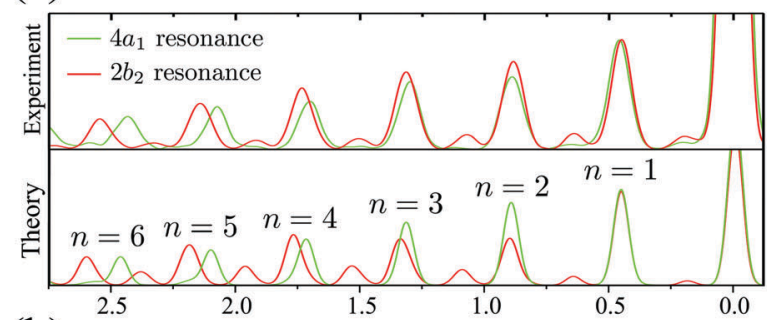

(b)

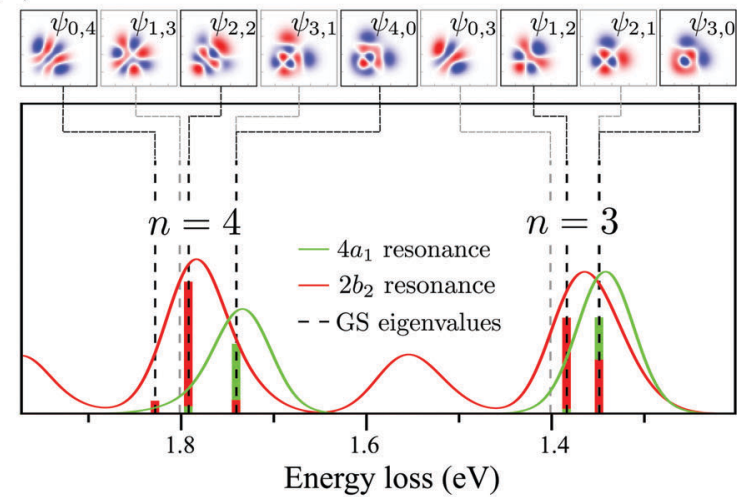

(c)

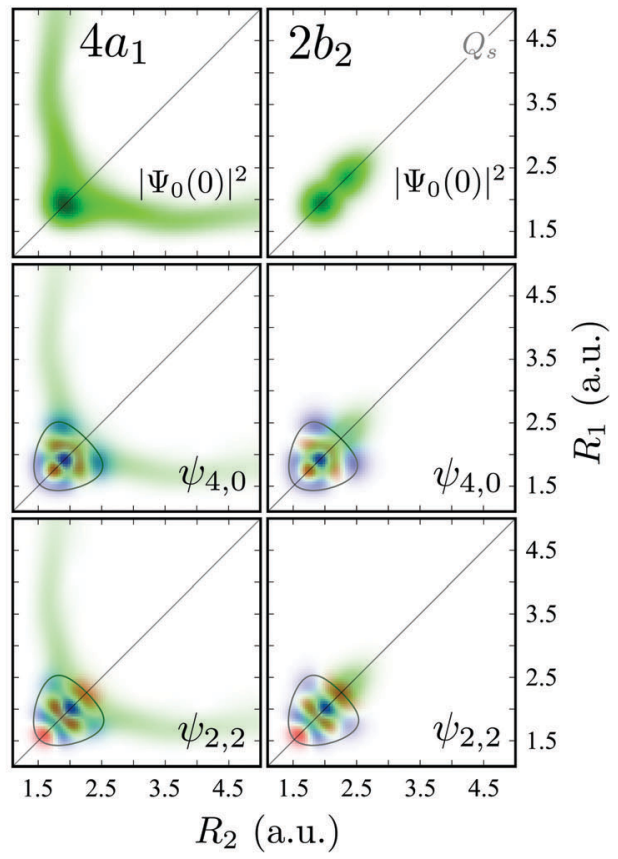

Fig. 5 (a) Comparison between experimental and theoretical RIXS at $4 a_{1}$ and $2 b_{2}$ resonances. The experimental curves are smoothed by spline interpolation. The detuning is the same as in Fig. 3. (b) A closer look at the theoretical RIXS spectra from panel (a). The ground state wave functions are shown on the top. The colored bars below the spectra are the Franck-Condon factors between the ground state wave functions and the core-excited wave packets $\left|\left\langle\psi_{n_{s}, n_{a}} \mid \Psi_{0}(0)\right\rangle\right|^{2}$. The gray dashed lines show the energies of the $\psi_{1,3}, \psi_{3,1}, \psi_{0,3}$ and $\psi_{2,1}$ states, which are forbidden in RIXS according to the selection rules (Section IIIC). (c) The overlap between the core-excited wave packet $\left|\Psi_{0}(0)\right|^{2}$ at the $4 \mathrm{a}_{1}$ and $2 \mathrm{~b}_{2}$ resonances and the ground state wave functions $\left(\psi_{n_{\mathrm{s}}, n_{\mathrm{a}}}\right)$. The contour lines in the wave function plots represent the classical turning curve of the ground state PES. The detuning is the same as in Fig. 3 .

come from the states $\psi_{n_{\mathrm{s}}, n_{\mathrm{a}}}$ with $n_{\mathrm{a}} \neq 0$. To explain this propensity rule, let us pay attention to the qualitatively different propagation dynamics of $\psi_{\mathrm{c}}(t)$ in the $4 \mathrm{a}_{1}$ and $2 \mathrm{~b}_{2}$ core-excited states (Fig. 4), which results in different spatial shapes of the integrated wave packet $\Psi_{0}(0)$ hence leading to changes in the FC amplitudes $\left|\left\langle\psi_{n_{s}, n_{\mathrm{a}}} \mid \Psi_{0}(0)\right\rangle\right|^{2}$ to the same final state $\psi_{n_{s}, n_{\mathrm{a}}}$.

Upon excitation at the $4 \mathrm{a}_{1}$ resonance, the wave packet propagates along the bonds $R_{1}$ and $R_{2}$, and within 7 fs the $\mathrm{H}$ atom is already 4 a.u. apart from the ${ }^{\bullet} \mathrm{OH}$ fragment, characterizing an ultrafast dissociation. The symmetric shape of the wave packet shows equal probability of the dissociation of either of the $\mathrm{OH}$ bonds. In the $2 \mathrm{~b}_{2}$ core-excited state, however, the wave packet is confined within the potential well and it bounces back and forth along the symmetric stretching coordinate $Q_{\mathrm{s}}$, which means that both $\mathrm{OH}$ bonds are excited coherently in this RIXS channel. The propensity rule is fully explained by Fig. 5c which compares $\left|\Psi_{0}(0)\right|^{2}(8)$ and its projection on the ground state wave functions $\psi_{4,0}$ and $\psi_{2,2}$ for the $4 a_{1}$ and $2 b_{2}$ intermediate core-excited states. Let us pay attention to the density lobes of the wave functions allocated near the eigenvalue contour lines, corresponding to the classical turning curves of the ground state potential, where the wave packet resides most of the time. ${ }^{18}$ The $4 \mathrm{a}_{1}$ core-excited wave packet overlaps better with $\psi_{4,0}$, which has density lobes along $R_{1}$ and $R_{2}$, rather than with $\psi_{2,2}$ the density lobes of which lie along $Q_{\mathrm{s}}$ (Fig. 5c). At the $2 \mathrm{~b}_{2}$ resonance, in contrast, the wave packet overlaps poorly with $\psi_{4,0}$ and perfectly with $\psi_{2,2}$ (Fig. 5c, right-hand side). This phenomenon results in an approximate selection rule, or propensity rule, in contrast to the strict symmetry selection rules (Section IIID). We will see in the following Section IVC that the propensity rule for the RIXS at the $4 \mathrm{a}_{1}$ resonance is violated for the large positive detuning, where the population of the state $\psi_{n_{\mathrm{s}}, 2}$ becomes significant.

There is an alternative explanation for why $\psi_{4,0}$ overlaps much better with the dissociative core-excited wave packet rather than with the $2 b_{2}$ one. Due to the degeneracy of the vibrational states $\psi_{n, 0}$ and $\psi_{n-1,1}$ (for $n \geq 3$ ) one can use on the same footing the localized states $\psi_{R_{1}}$ and $\psi_{R_{2}}$ defined in eqn (5) and displayed in Fig. 2c. The strong bond localization of these states explains the discussed propensity rules for the $\psi_{n, 0}$ levels in the $4 \mathrm{a}_{1}$ and $2 \mathrm{~b}_{2}$ spectra.

It is important to emphasize that the spatial shape of $\Psi_{0}(0)$ is defined by the shape of the PES, which is in general different for different core-excited states in a molecule. Due to this, the propensity rules can be exploited for selective probing of the ground state potential of multimode systems, as it has been recently demonstrated. ${ }^{40}$

\section{Wave packet dynamics controlled by the excitation energy detuning}

It is well established that the shape of a RIXS spectrum is very sensitive to the dynamics of the nuclear wave packet in the core-excited PES, which depends inherently on the PES shape. However, there is also a possibility to manipulate the core- 
excited wave packet dynamics within the selected PES by controlling the scattering duration ${ }^{18,50}$

$$
\tau=\frac{1}{\sqrt{\Omega^{2}+\Gamma^{2}}},
$$

by varying the excitation energy detuning $\Omega$ (15) from the resonance. For example, it allows manipulating the wave packet distribution in the core-excited state for advanced mapping of the final state potential in resonant X-ray scattering. ${ }^{32,77}$ To understand the experimental dependence of the $\mathrm{H}_{2} \mathrm{O}$ RIXS spectrum on detuning, we performed correspondent simulations of the RIXS cross section at the $4 a_{1}, 2 b_{2}$, and $2 b_{1}$ resonances.

1. Detuning around the $2 b_{1}$ resonance. Six excitation energies around the $2 b_{1}$ resonance were studied theoretically. The experimental spectrum, obtained at $\Omega=+0.2 \mathrm{eV}$, gives a good qualitative

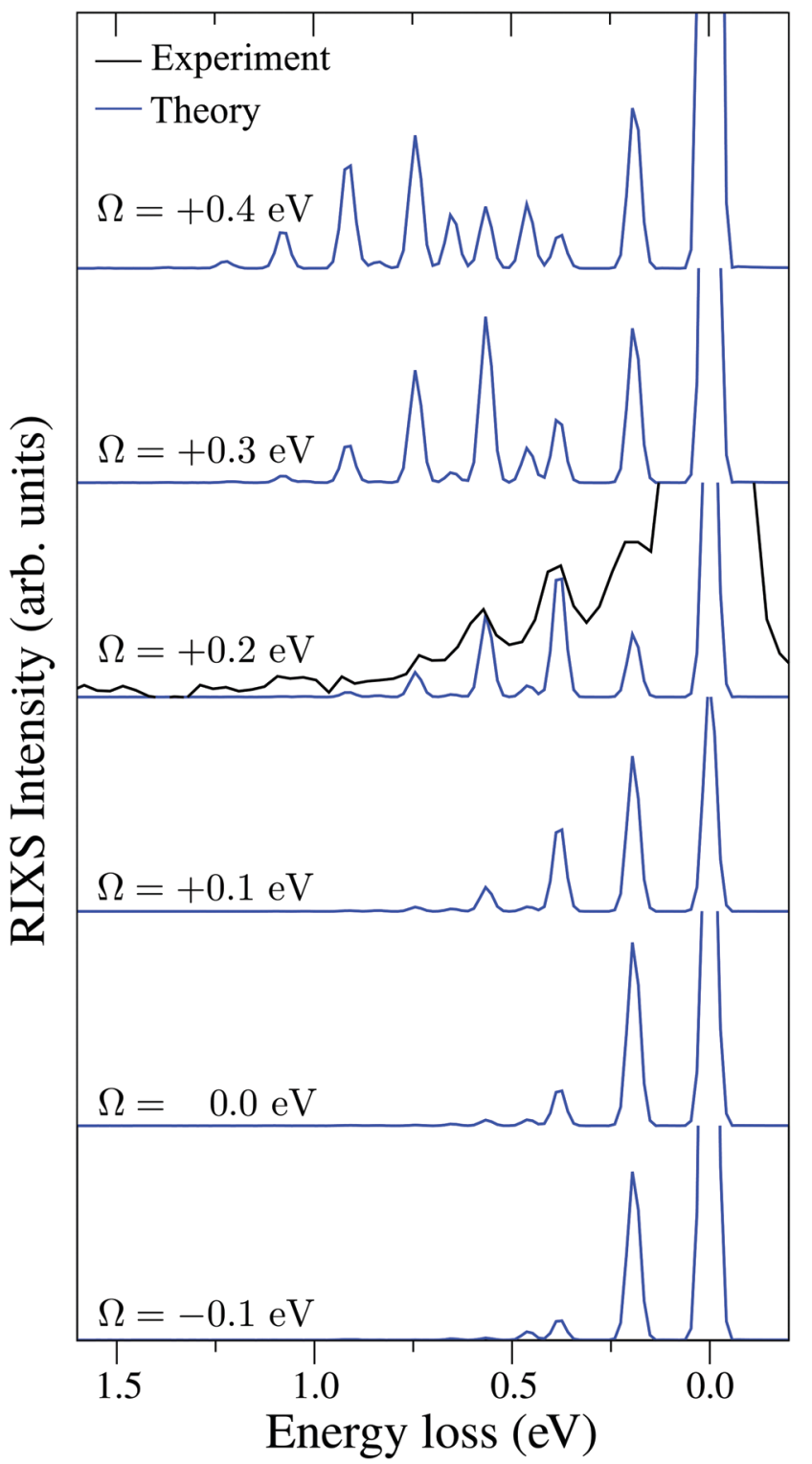

Fig. 6 Experimental RIXS at the $2 \mathrm{~b}_{1}$ resonance for $\Omega=+0.2 \mathrm{eV}$ and theoretical RIXS at various detuning values $\Omega$. agreement with the theory. The results (Fig. 6) show a shortening of the vibrational progression in RIXS, the so-called vibrational collapse, ${ }^{78,79}$ when the frequency $\omega$ is tuned below the top of the XAS resonance and the scattering duration (16) becomes small. With excitation energy around the top of the resonance $(\Omega=0.0 \ldots+0.2 \mathrm{eV})$ the vibrational progression becomes longer, but still consists of only bending mode quanta, as it has been discussed previously. However, as we detune more $(\Omega=+0.4 \mathrm{eV})$ the admixture of the stretching vibrational mode becomes visible in the spectrum around $\omega-\omega^{\prime} \approx 0.45 \mathrm{eV}$. This reflects the fact that even though the minima and the shape of the $2 \mathrm{D}$ stretching PES near the bottom are almost the same for the ground and the $2 b_{1}$ core-excited state, the shape of these PESs becomes rather different for higher vibrational states. Therefore, for large positive values of detuning the mode selectivity vanishes for this resonance.

It is necessary to address the peculiar behavior observed for the peak intensities in the theoretical model, showing a

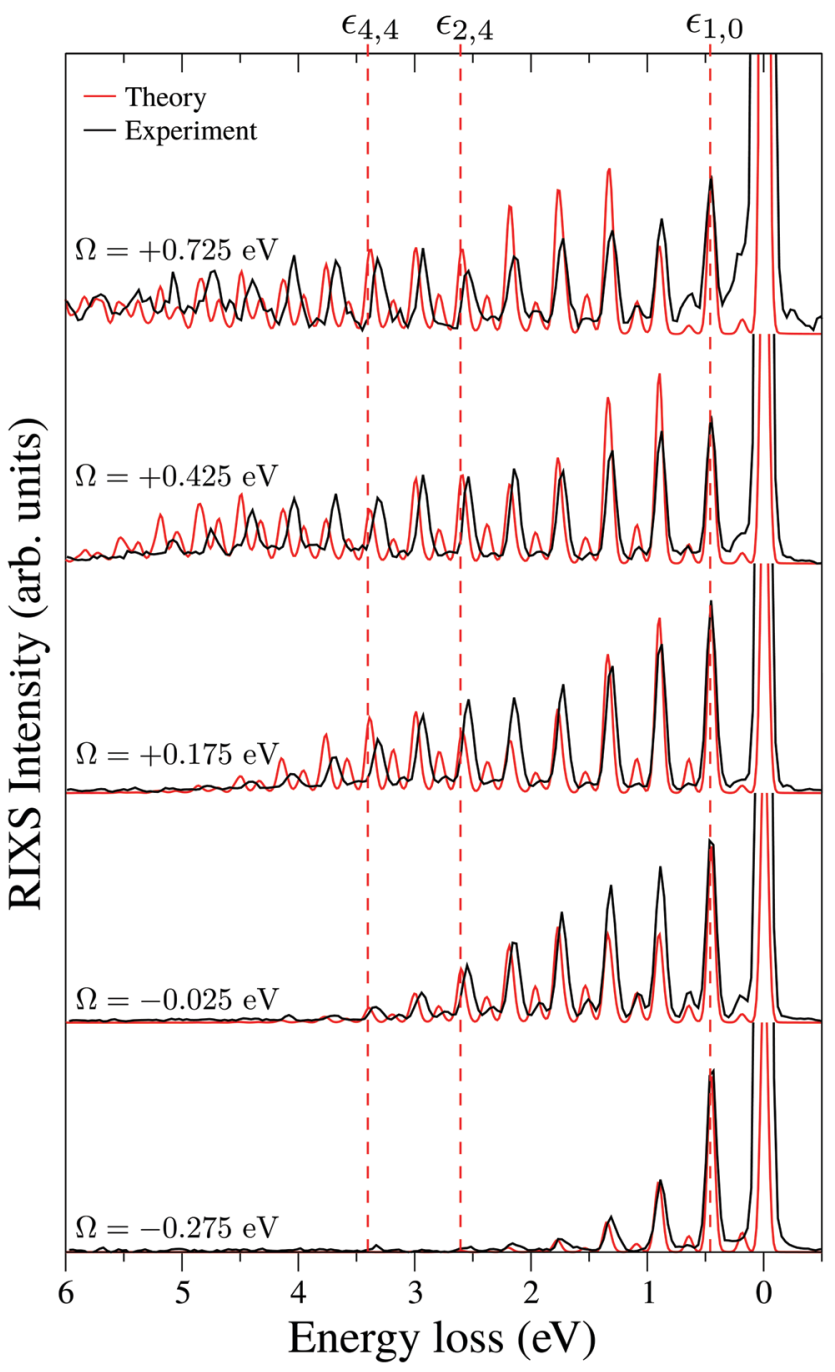

Fig. 7 Theoretical and experimental RIXS at the $2 b_{2}$ resonance for various detuning values $\Omega$. The dashed lines show the energies of the $\psi_{1,0}, \psi_{2,4}$ and $\psi_{4,4}$ states at $\varepsilon_{1,0}=0.45, \varepsilon_{2,4}=2.61$ and $\varepsilon_{4,4}=3.35 \mathrm{eV}$, respectively. 
non-monotonous intensity distribution. It arises due to the selfabsorption effect, which is taken into account approximately in our theoretical model (12), and it is clearly overestimated for the second vibrational peak in the RIXS at the $2 b_{1}$ resonance.

2. Detuning around the $2 \mathbf{b}_{2}$ resonance. Fig. 7 collects the experimental and theoretical RIXS spectra near the $2 b_{2}$ resonance for five different values of detuning: $\Omega=-0.275,-0.025$, $+0.175,+0.425$ and $+0.725 \mathrm{eV}$. One can notice the vibrational collapse $^{80}$ trend for large detuning below the resonance. Let us note that the vibrational collapse also occurs for large positive detuning. However, this happens for much larger positive values of $\Omega$ due to the asymmetry of the XAS vibrational structure with respect to the vertical transition.

To explain in more detail the shortening of the vibrational progression on the way from positive to negative detuning lets us compare the frequency of sign-changing oscillations of the wave packet $\Psi_{0}(0)$ and the eigenstate $\psi_{4,4}$ along $Q_{\mathrm{s}}$ (see Fig. 8). This frequency is proportional to the number of nodes along the antisymmetric mode. The number of nodes is 5 for $\psi_{4,4}$, while $\Psi_{0}(0)$ has 6,2 and 3 nodes for $\Omega=+0.725,+0.175$ and $-0.025 \mathrm{eV}$, respectively. The growing mismatch of the frequency of the sign-changing oscillations (number of nodes) of $\psi_{4,4}$ and $\Psi_{0}(0)$ explains the strong suppression of the peak $\varepsilon_{4,4}$ on the way from $\Omega=+0.725$ to $-0.025 \mathrm{eV}$ seen in Fig. 7 .

One should note that each peak of the spectra shown in Fig. 7 has an underlying fine structure (see Table 1), caused by quasi-degenerated stretching modes, which is, however, hidden by the total experimental broadening $\Delta=0.098 \mathrm{eV}$. One can see that each bending peak $\left(\nu=0 \rightarrow \nu_{\mathrm{f}}=1\right)$ copies the fine structure of the parent stretching peak because the coupling between the bending and stretching motions is neglected.

Fig. 7 displays some shift between the theoretical and experimental RIXS spectra for energy loss $\omega-\omega^{\prime}>1 \mathrm{eV}$, which deserves a special comment. The potentials used in the simulations are obtained directly from the $a b$ initio electronic structure calculations, the accuracy of which drops down as the geometry becomes distorted. Due to this fact, the potential energy surface steepness is overestimated at large $R_{1}$ and $R_{2}$, resulting in a slightly larger vibrational frequency as compared to one observed in the experiment (Table 1). In previous studies, ${ }^{81}$ the most accurate potentials were obtained by combining $a b$ initio and experimental data, where the computed potential was fitted with the vibrational energies measured in IR experiments. Another possible reason for the observed shift is a breakdown of the $2 \mathrm{D}+1 \mathrm{D}$ approximation, resulting in a shift of the center of gravity of the stretching peak due to possible redistribution of the FC factors within the $n$th group. One can also observe some overestimation of the intensity of the bending peaks in the region $\omega-\omega^{\prime}>3 \mathrm{eV}$ for $\Omega=+0.425$ and $+0.725 \mathrm{eV}$, which can also be attributed to the limitation of the $2 \mathrm{D}+1 \mathrm{D}$ model. The $2 \mathrm{D}+1 \mathrm{D}$ approximation may breakdown for higher vibrational states $\left(\omega-\omega^{\prime}>2 \mathrm{eV}\right)$, where $R_{1}$ and $R_{2}$ are large and the bond angle may deviate significantly from the equilibrium.

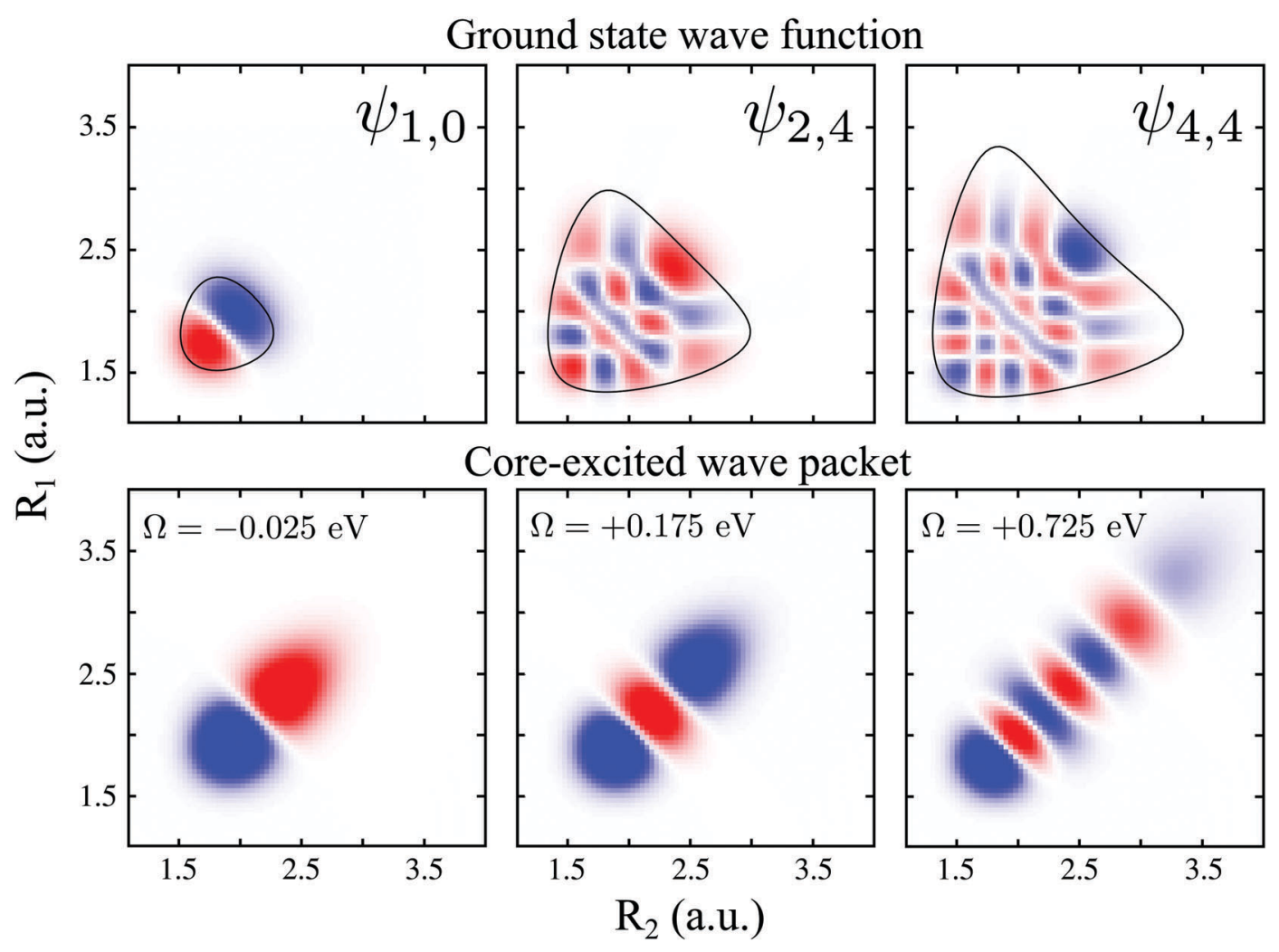

Fig. 8 Upper panel: $\psi_{1,0}, \psi_{2,4}$ and $\psi_{4,4}$ ground state wave functions. The contour around the wave functions represents the edges of the PES (classical turning curves). Lower panel: real part of the $2 \mathrm{~b}_{2}$ core-excited wave packet $\Psi_{0}(0)$ giving by eqn (10), for detuning values $\Omega=-0.025,+0.175$ and +0.725 eV . 
3. Detuning around the $4 a_{1}$ resonance. The RIXS spectra measured at different excitation energies near the dissociative $4 \mathrm{a}_{1}$ core-excited state can be used effectively for probing the ground state stretching potential. The reason for this is the propensity rule which cleans up the spectra from many quasidegenerated vibrational states (Section IVB). The experimental and theoretical RIXS spectra at the $4 \mathrm{a}_{1}$ resonance are presented in Fig. 9, for detuning values $\Omega=-1.20,-0.95,-0.70,-0.40$, $-0.15,+0.05,+0.30,+0.60,+0.78,+1.03$ and $+1.28 \mathrm{eV}$ and one can notice a remarkably good agreement between theory and experiment. In contrast to the RIXS at the $2 b_{2}$ resonance (Fig. 7), a rather long vibrational progression is already seen for $\Omega=-0.70 \mathrm{eV}$. The reason for this is the core-excited dynamics (Fig. 4) which leads to qualitatively different vibrational structures below the top of the $2 \mathrm{~b}_{2}$ and $4 \mathrm{a}_{1}$ XAS profiles (Fig. 3a). Due to the broad continuum for the dissociative core-excited state and only few vibrational states in the $2 b_{2}$ case, the collapse of the vibrational structure ${ }^{78,79}$ is manifested in these states for $\Omega<-1 \mathrm{eV}$ and $\Omega<-0.2 \mathrm{eV}$, respectively.

The RIXS at the $4 \mathrm{a}_{1}$ resonance (Fig. 9) shows that the propensity rule, which allows only the $\psi_{n, 0}$ to be excited, is valid in the region $\Omega<0.6 \mathrm{eV}$. This rule is violated for higher detuning where one can observe a doubling of the RIXS resonances in the theoretical spectra, and hence the loss of selectivity. The loss of selectivity is also observed in the experimental spectra, however, due to a low signal-to-noise ratio the doubling of peaks is not sharply seen. To shed light on this effect, let us look at Fig. 10, which compares the spectra for $\Omega=+0.30$ and $\Omega=+1.03 \mathrm{eV}$. At the lower detuning the intensity of the $\psi_{4,2}$ vibrational state is strongly suppressed, as it is expected according to the propensity rule. However, this "forbidden" peak arises for the larger detuning. The reason for this is the extension of the wave packet $\Psi_{0}(0)$ along $Q_{\mathrm{s}} \approx R_{2}-R_{1}$. In this case, $\Psi_{0}(0)$ becomes more similar to the shape of the $\psi_{4,2}$ wave function and the intensity of the "forbidden" peak $\left|\left\langle\psi_{4,2} \mid \Psi_{0}(0)\right\rangle\right|^{2}$ increases. The breakdown of the propensity rule for $\Omega>0.6 \mathrm{eV}$ is seen in the region $\omega-\omega^{\prime}>2 \mathrm{eV}$ (Fig. 9). Therefore, this shows how the excitation energy constitutes an extra tool to control the previously reported gating effect. ${ }^{40}$

\section{Role of isotopic substitution}

To obtain a complete picture of the RIXS spectrum of water, we study here the isotopically substituted water molecules, $\mathrm{D}_{2} \mathrm{O}$ and HDO. Replacing the hydrogen atom by deuterium leads to a large effect in the vibrational spectrum of RIXS. The effect of the isotopic substitution $\mathrm{H}_{2} \mathrm{O} \rightarrow \mathrm{D}_{2} \mathrm{O}$ is twofold: the vibrational energy spacing is reduced in the RIXS spectrum and the nuclear dynamics becomes slower. The isotopic substitution $\mathrm{H}_{2} \mathrm{O} \rightarrow \mathrm{HDO}$, besides changing the vibrational frequencies and the dynamics, also breaks the symmetry of the molecule, resulting in the

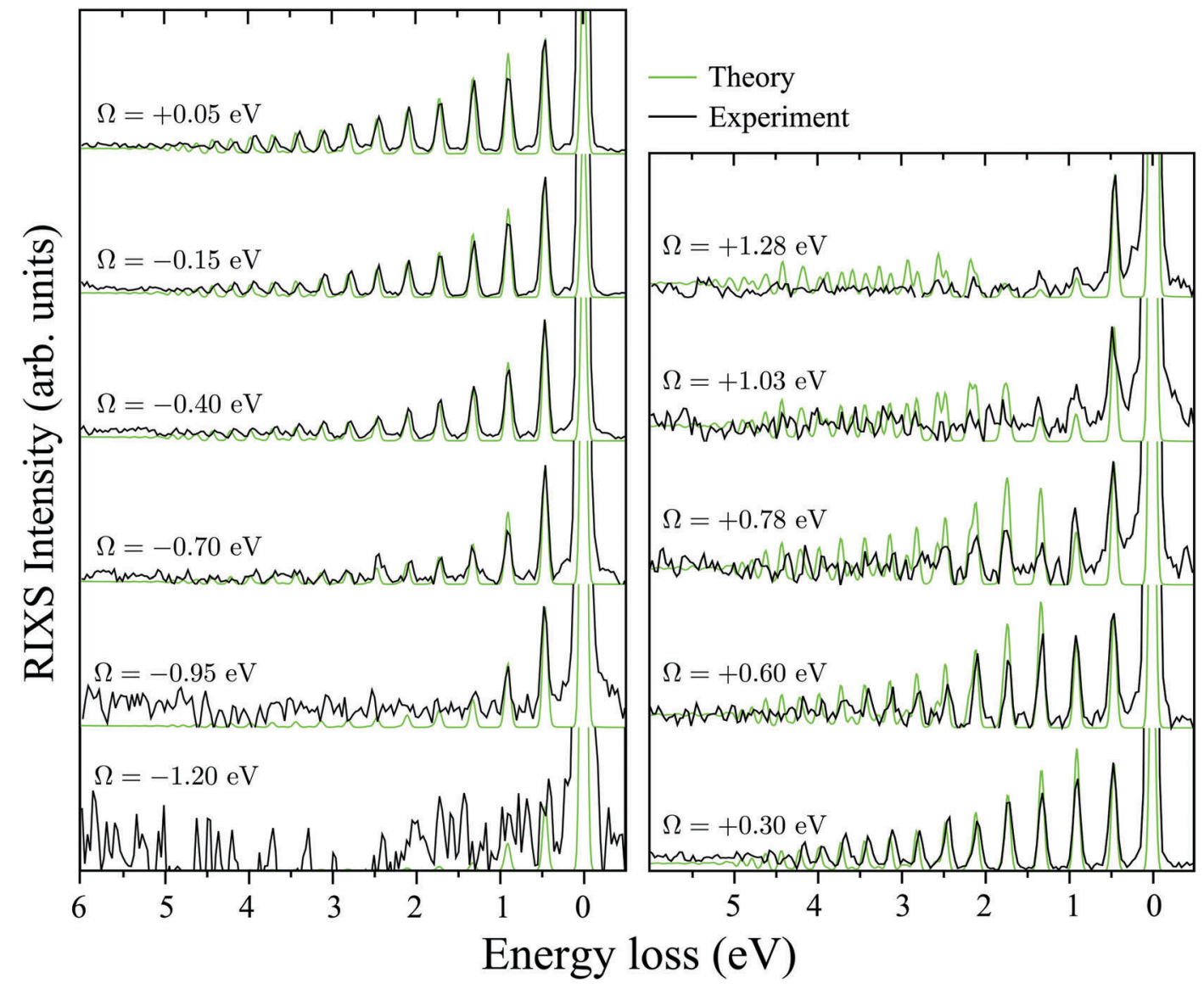

Fig. 9 Theoretical and experimental RIXS at $4 \mathrm{a}_{1}$ resonance for different values of detuning $\Omega$. 


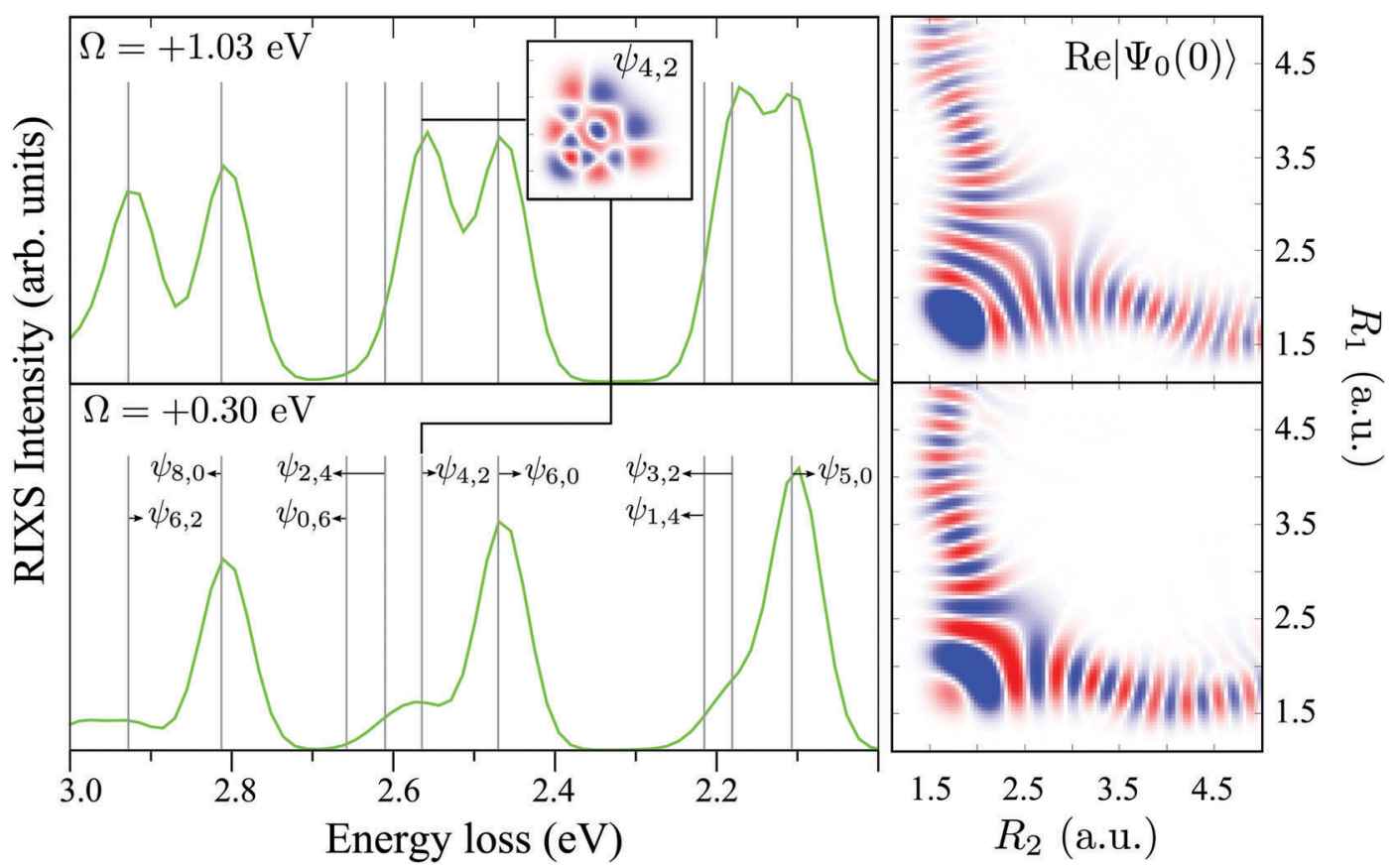

Fig. 10 Details of the RIXS at the $4 \mathrm{a}_{1}$ resonance for different detuning values $\Omega$. The propensity rule, seen near the top of the XAS $(\Omega=+0.30 \mathrm{eV})$, is violated at large detuning $(\Omega=+1.03 \mathrm{eV})$ due to the change in the shape of the core-excited wave packet (right-hand side plots). The vertical lines show the energy position of the ground state wave functions.

localization of the vibrational modes on either of the bonds (see, however, Section I and ref. 41). Since the stretching modes are responsible for the main spectral features, the bending mode is fully neglected here for clarity. For the same reason, we omit here the discussion of RIXS via the $2 \mathrm{~b}_{1}$ state, where only the bending mode is excited (Fig. 3b). The PESs used to describe the ground and core-excited states of $\mathrm{D}_{2} \mathrm{O}$ and $\mathrm{HDO}$ are the same as for $\mathrm{H}_{2} \mathrm{O}$ (Fig. 1), since the atomic masses affect mainly the nuclear dynamics. One should mention that different isotopes may couple the electronic potentials slightly via weak vibronic coupling.

It is helpful to analyze the ground state vibrational properties of $\mathrm{D}_{2} \mathrm{O}$ and HDO. The numerical solution of the 2D eigenvalue problem gives the vibrational spectrum (Table 2) and the respective wave functions (Fig. 11). Fig. 11 shows that for HDO, the vibrational wave functions are localized along the bonds $R_{1}(\mathrm{OH})$ or $R_{2}(\mathrm{DO})$, due to the breaking of the molecular symmetry. Therefore, in contrast to the $\psi_{n_{\mathrm{s}}, n_{\mathrm{a}}}$ notation used for $\mathrm{H}_{2} \mathrm{O}$ and $\mathrm{D}_{2} \mathrm{O}$, we assign the localized vibrational states $\psi_{n_{\mathrm{D}}, n_{\mathrm{H}}}$ of HDO using the quantum numbers $n_{\mathrm{D}}$ and $n_{\mathrm{H}}$, as well as the group number $n=n_{\mathrm{D}}+n_{\mathrm{H}}$. Table 2 also shows the violation of the grouping of the vibrational states of HDO within the group $n=n_{\mathrm{D}}+n_{\mathrm{H}}$. For example, at $n=3$ the last vibrational level should be the $\psi_{0,3}$ level (as in $\mathrm{H}_{2} \mathrm{O}$ and $\mathrm{D}_{2} \mathrm{O}$ ), but the $\psi_{4,0}$ level is seen instead. The vibrational wave functions of $\mathrm{H}_{2} \mathrm{O}$ and $\mathrm{D}_{2} \mathrm{O}$ (Fig. 11) are similar, as the molecular symmetry is preserved upon the isotopic substitution. However, the wave functions of $\mathrm{D}_{2} \mathrm{O}$ have slightly smaller size since they are localized closer to the PES bottom due to the smaller DO vibrational frequency $\left(\omega_{\mathrm{DO}}=0.32 \mathrm{eV}\right.$ and $\left.\omega_{\mathrm{OH}}=0.45 \mathrm{eV}\right)$.
Table 2 Ground state stretching vibrational energies of the $\mathrm{H}_{2} \mathrm{O}, \mathrm{D}_{2} \mathrm{O}$ and HDO molecules. The states which do not follow the $n$ grouping order are highlighted in bold. The rest of the notations are the same as in Table 1

\begin{tabular}{lllllll}
\hline$n$ & $N$ & $\left(n_{\mathrm{s}}, n_{\mathrm{a}}\right)$ & $\mathrm{H}_{2} \mathrm{O}$ & $\mathrm{D}_{2} \mathrm{O}$ & $\left(n_{\mathrm{D}}, n_{\mathrm{H}}\right)$ & $\mathrm{HDO}$ \\
\hline 1 & 1 & $(1,0)$ & 0.4588 & 0.3361 & $(1,0)$ & 0.3403 \\
& 2 & $(0,1)$ & 0.4680 & 0.3450 & $(0,1)$ & 0.4637 \\
& & & & & & \\
& 3 & $(2,0)$ & 0.9003 & 0.6637 & $(2,0)$ & 0.6678 \\
& 4 & $(1,1)$ & 0.9037 & 0.6683 & $(1,1)$ & 0.8034 \\
& 5 & $(0,2)$ & 0.9295 & 0.6853 & $(0,2)$ & 0.9043 \\
& & & & & & \\
3 & 6 & $(3,0)$ & 1.3222 & 0.9810 & $(3,0)$ & 0.9837 \\
& 7 & $(2,1)$ & 1.3229 & 0.9826 & $(2,1)$ & 1.1303 \\
& 8 & $(1,2)$ & 1.3581 & 1.0025 & $(1,2)$ & 1.2433 \\
& 9 & $(0,3)$ & 1.3763 & 1.0186 & $(4,0)$ & 1.2892 \\
& & & & & & \\
4 & 10 & $(4,0)$ & 1.7241 & 1.2875 & $(0,3)$ & 1.3252 \\
& 11 & $(3,1)$ & 1.7242 & 1.2878 & $(3,1)$ & 1.4455 \\
& 12 & $(2,2)$ & 1.7769 & 1.3150 & $(2,2)$ & 1.5695 \\
& 13 & $(1,3)$ & 1.7869 & 1.3260 & $(5,0)$ & 1.5846 \\
& 14 & $(0,4)$ & 1.8141 & 1.3461 & $(1,3)$ & 1.6634 \\
& & & & & & \\
5 & 15 & $(5,0)$ & 2.1070 & 1.5833 & $(0,4)$ & 1.7274 \\
& 16 & $(4,1)$ & 2.1070 & 1.5834 & $(4,1)$ & 1.7504 \\
& 17 & $(3,2)$ & 2.1807 & 1.6214 & $(6,0)$ & 1.8703 \\
& 18 & $(2,3)$ & 2.1841 & 1.6272 & $(3,2)$ & 1.8841 \\
& 19 & $(1,4)$ & 2.2153 & 1.6468 & $(2,3)$ & 1.9888 \\
& 20 & $(0,5)$ & 2.2408 & 1.6675 & $(5,1)$ & 2.0451 \\
& & & & & & \\
\hline & & & & & &
\end{tabular}

The XAS of $\mathrm{D}_{2} \mathrm{O}$ and HDO are presented in Fig. 12a, and a quick comparison with spectrum of $\mathrm{H}_{2} \mathrm{O}$ (Fig. 3a) does not show significant changes in the spectral profile, except for the slight shift of the XAS maxima: $\omega_{4 \mathrm{a}_{1}}^{\mathrm{XAS}}=534.11$ and $\omega_{2 \mathrm{~b}_{2}}^{\mathrm{XAS}}=$ $535.90 \mathrm{eV}$ for HDO, and $\omega_{4 \mathrm{a}_{1}}^{\mathrm{XAS}}=534.15$ and $\omega_{2 \mathrm{~b}_{2}}^{\mathrm{XAS}}=536.03 \mathrm{eV}$ 


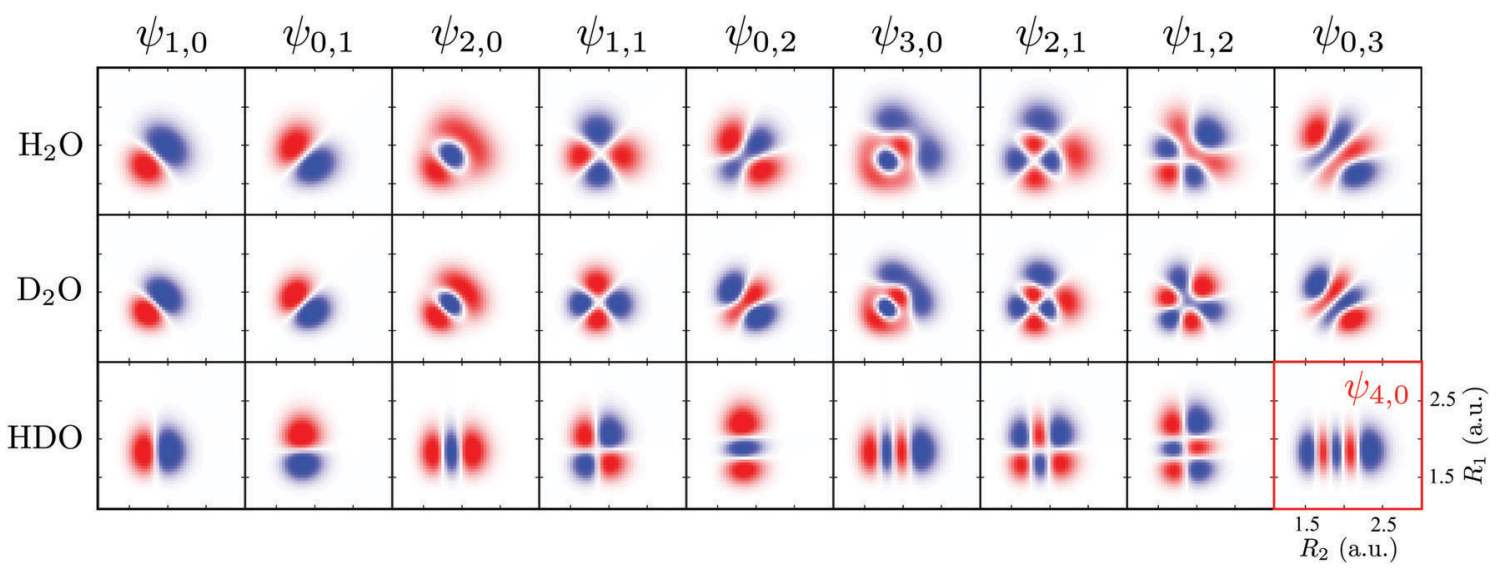

Fig. 11 Wave functions of the ground state of $\mathrm{H}_{2} \mathrm{O}, \mathrm{D}_{2} \mathrm{O}\left(\psi_{\mathrm{n}_{\mathrm{s}}, n_{\mathrm{a}}}\right)$ and $\mathrm{HDO}\left(\psi_{n_{\mathrm{D}}, n_{\mathrm{H}}}\right)$. In the HDO, $R_{1}$ represents the O-H bond and $R_{2}$ the D-O bond. All wave functions are in the same scale.

(a)

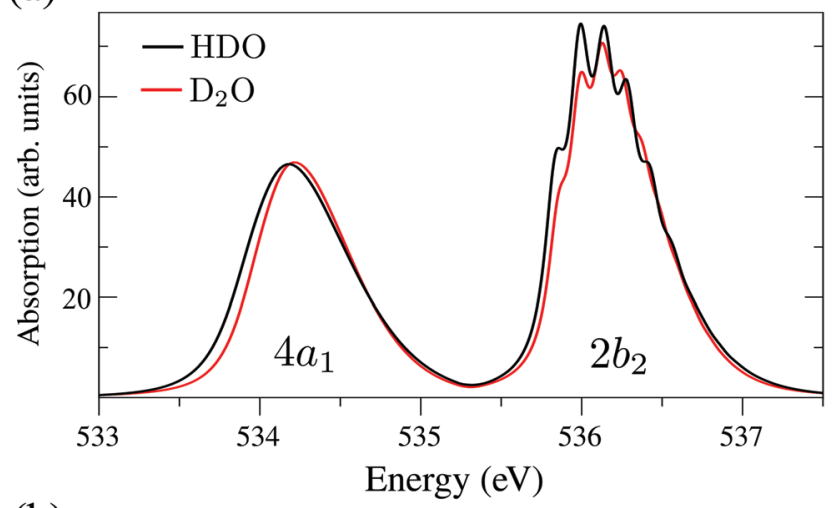

(b)

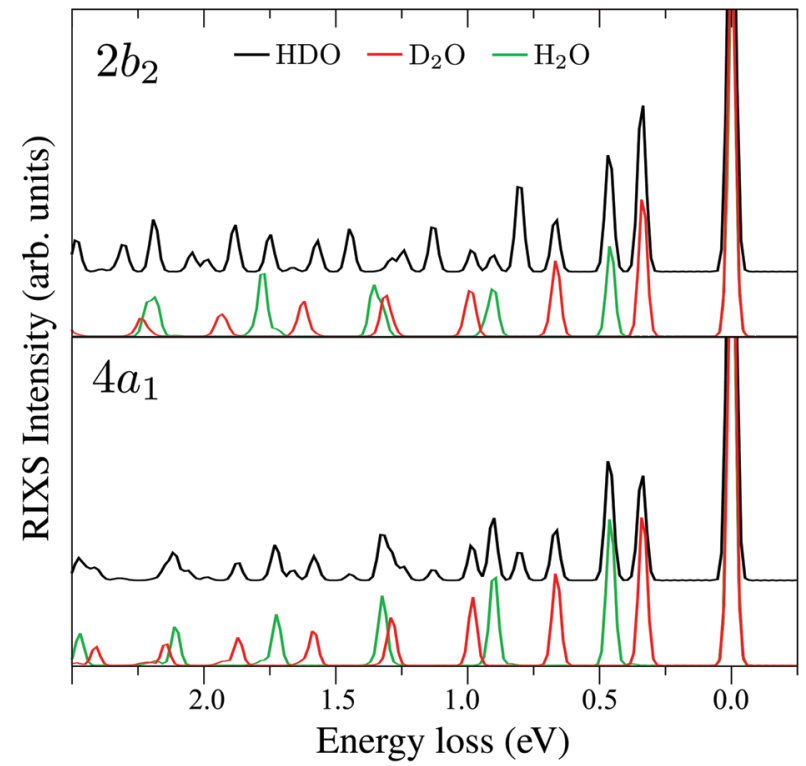

Fig. 12 (a) Theoretical X-ray absorption spectra of $\mathrm{HDO}$ and $\mathrm{D}_{2} \mathrm{O}$. (b) Theoretical RIXS at the $4 a_{1}$ and $2 b_{2}$ resonances for $\mathrm{H}_{2} \mathrm{O}, \mathrm{D}_{2} \mathrm{O}$ and HDO $\left(\Omega_{2 \mathrm{~b}_{2}}=-0.025 \mathrm{eV}\right.$ and $\left.\Omega_{4 \mathrm{a}_{1}}=+0.05 \mathrm{eV}\right)$.

for $\mathrm{D}_{2} \mathrm{O}$. Considering the excitation energy at the top of absorption resonance $(\Omega=0)$, the RIXS spectra of $\mathrm{D}_{2} \mathrm{O}$ and
HDO at the $4 a_{1}$ and $2 b_{2}$ resonances were simulated, and are presented in Fig. 12b along with the RIXS spectrum of $\mathrm{H}_{2} \mathrm{O}$ for comparison. As expected, the RIXS spectra of $\mathrm{H}_{2} \mathrm{O}$ and $\mathrm{D}_{2} \mathrm{O}$ have similar vibrational profiles, but due to the different $\mathrm{OH}$ and OD vibrational frequencies, the spectra are shifted, at both $4 \mathrm{a}_{1}$ and $2 \mathrm{~b}_{2}$ resonances. However, the HDO RIXS presents a more interesting profile. From Fig. 12b, one can see that for $\omega-\omega^{\prime}<1 \mathrm{eV}$, the HDO RIXS contains both $\mathrm{H}_{2} \mathrm{O}$ and $\mathrm{D}_{2} \mathrm{O}$ resonances, since both $\mathrm{OH}$ and $\mathrm{OD}$ vibrations are present. Moreover, due to the symmetry breaking, the selection rules discussed in Section IIID are not valid anymore for the HDO molecule. Therefore, transitions to all states are allowed and the number of vibrational resonances in the RIXS spectrum increases (Fig. 13). However, the propensity rule is yet preserved, as observed for $\omega-\omega^{\prime}>1.5 \mathrm{eV}$. The real part of the core-excited wave packet in the $4 \mathrm{a}_{1}$ and $2 \mathrm{~b}_{2}$ states (Fig. 13b) shows that even though the symmetry is broken in HDO and the wave functions are localized in the ground state, the coreexcited wave packet is delocalized, as in $\mathrm{H}_{2} \mathrm{O}$ (Fig. 5c), preserving the propensity rule. A deeper insight into this mode delocalization in core-excited states can be found elsewhere. ${ }^{41}$

\section{E. "Atomic" peak}

Following core excitation at the $4 \mathrm{a}_{1}$ resonance, the ultrafast dissociation of an $\mathrm{OH}$ bond of the water molecule is observed. This can be observed from the analysis of the time-dependent core-excited wave packet $\psi_{\mathrm{c}}(t)$, which propagates far away from the equilibrium during the lifetime of the core-excited state (Fig. 4). The wave packet $\psi_{\mathrm{c}}(t)$ on the way to the asymptotic region of the dissociation decays continuously to the ground state. The continuum-bound decay near the equilibrium forms the so-called molecular band ( $5 \mathrm{eV} \gtrsim \omega-\omega^{\prime} \geq 0$, see Fig. 14). When the wave packet approaches the region of dissociation, the dominant process is the continuum-continuum decay, which is nothing else than the decay transitions in the fragment of dissociation ${ }^{\circ} \mathrm{OH}$. These decay transitions form the so-called "atomic" peak, ${ }^{42,43,82}$ which in the case of the ${ }^{\bullet} \mathrm{OH}$ fragment has a vibrational structure defined by the $\mathrm{OH}$ vibrational frequency. The RIXS spectra at the $4 \mathrm{a}_{1}$ resonance for $\mathrm{H}_{2} \mathrm{O}, \mathrm{D}_{2} \mathrm{O}$ and $\mathrm{HDO}$ are 
(a)

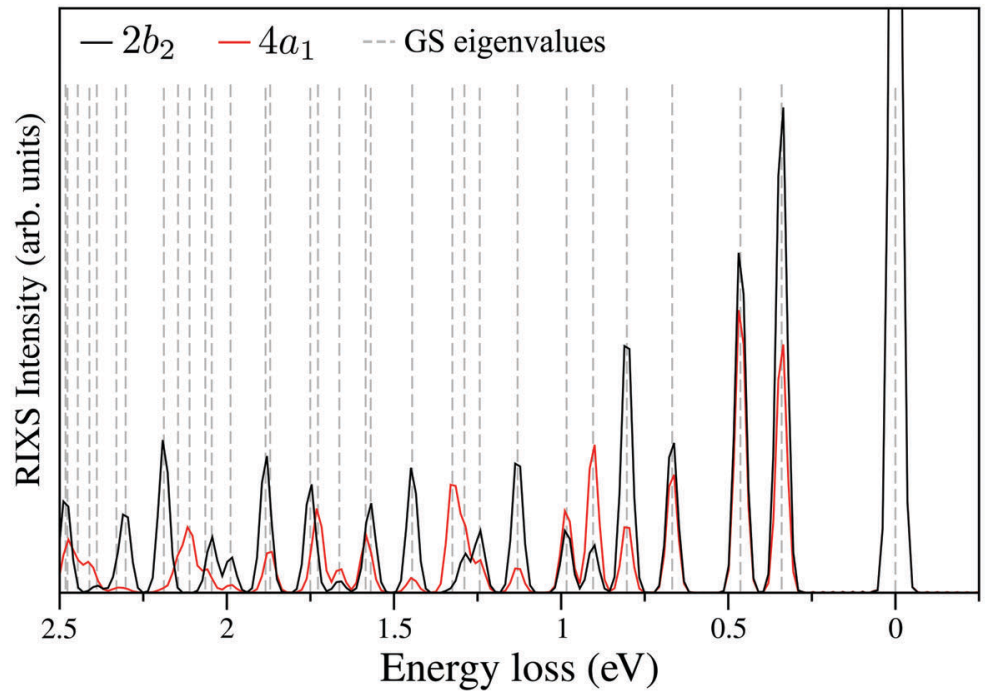

(b)

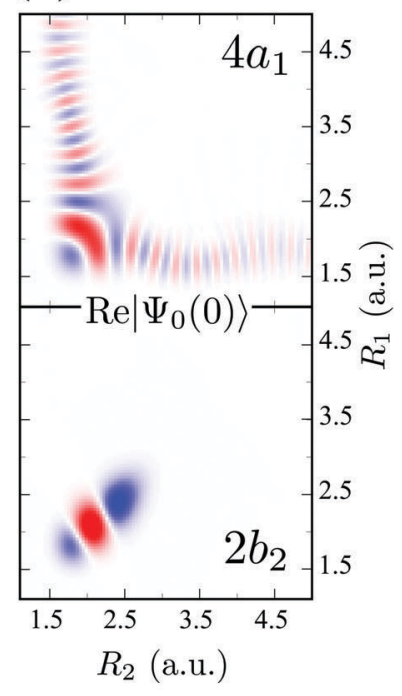

Fig. 13 (a) Theoretical HDO RIXS at the $4 a_{1}$ and $2 b_{2}$ resonances. The ground state eigenvalues are shown by the dashed lines and their assignment follows the energy order of Table 2. (b) Real part of the core-excited wave packet $\Psi_{0}(0)$ at $4 \mathrm{a}_{1}$ and $2 \mathrm{~b}_{2}$ resonances for HDO.

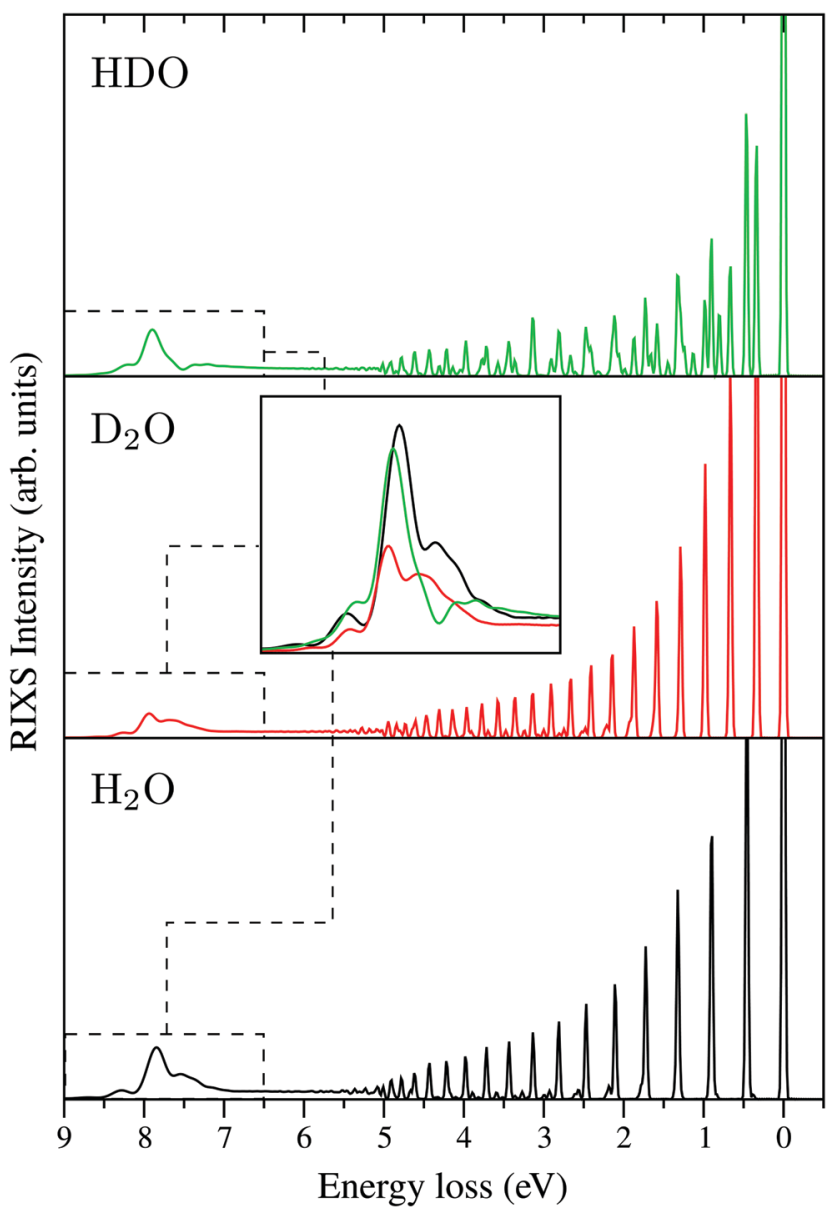

Fig. 14 RIXS spectra at the $4 \mathrm{a}_{1}$ resonance for $\mathrm{H}_{2} \mathrm{O}, \mathrm{D}_{2} \mathrm{O}$ and $\mathrm{HDO}$, which show the formation of the "atomic" peak at around $7.5 \mathrm{eV}$. The detuning is the same as used in Fig. 12b. All spectra are given at the same intensity scale. The "atomic" peak exhibits a vibrational structure as it is formed by the emission transition from the core-excited fragment of dissociation ${ }^{\bullet} \mathrm{OH}$. presented in Fig. 14, with the "atomic" peak shown at around $7.5 \mathrm{eV}$ of energy loss. The "atomic" peak of $\mathrm{D}_{2} \mathrm{O}$ has a smaller intensity than the $\mathrm{H}_{2} \mathrm{O}$ and $\mathrm{HDO}$ ones. As deuterium is heavier than hydrogen the dissociation $\mathrm{D}-\mathrm{O} \cdots \mathrm{D}$ is slower, and a smaller portion of the wave packet reaches the dissociation region during the short core-hole lifetime. The "atomic" peaks of $\mathrm{HDO}$ and $\mathrm{H}_{2} \mathrm{O}$ are comparable, showing only a slightly different structure. This happens because the "atomic" peak of HDO is composed by the $\mathrm{H} \cdots \mathrm{DO}$ and $\mathrm{D} \cdots \mathrm{HO}$ fragmentation.

The isotope sensitivity of the "atomic" peak in the presented RIXS spectra sheds light on its dynamical origin. This is important in order to understand the electronically-inelastic scattering spectrum of gas-phase water. ${ }^{83}$ This peak cannot be isolated in the total experimental RIXS spectrum, due to overlap with the $\left|1 \mathrm{~b}_{1}{ }^{-1} 4 \mathrm{a}_{1}{ }^{1}\right\rangle$ final state, which has the same dissociation limit as the ground state. Our simulations, however, can help to understand the recent experimental results, ${ }^{83}$ where a splitting of the inelastic band of the $\left|1 b_{1}{ }^{-1} 4 a_{1}{ }^{1}\right\rangle$ final state is observed and tentatively referred to the nuclear dynamics effect. Our analysis assures that the $\Omega$-dependence of the observed doublet ${ }^{83}$ is due to the presence of the "atomic" peak which is very sensitive to the dissociation dynamics defined by the scattering duration and mass of $\mathrm{OH}$ or $\mathrm{OD}$.

\section{Conclusions}

Using ultra-high resolution X-ray spectroscopy, state-of-the-art $a b$ initio potential energy surfaces and strict nuclear wave packet propagation calculations, we carried out a complete elucidation of the electronically-elastic RIXS spectra of gasphase water. Based on an excellent agreement between theory and experiment, several conclusions, of detailed as well as general character, could be drawn. The simulations show rather 
complicated structures of the vibrational spectrum of the ground state due to strong anharmonicity and strong mode coupling. We demonstrated through a fine analysis of the wave packet dynamics that RIXS offers a simplification of the challenging coupled-mode dynamics problem in $\mathrm{H}_{2} \mathrm{O}$ since scattering through different core-excited states acts as a filter to probe different vibrational progressions, thus probing different parts of the multi-dimensional ground state PES. Close to the respective top of resonance, RIXS at the $2 b_{1}$ resonance excites solely the bending vibrational mode, while at the $4 \mathrm{a}_{1}$ resonance mainly the symmetric stretching mode is excited. In RIXS process through the $2 b_{2}$ core-excited state, the bending mode and a mixture of the two stretching vibrational modes are excited.

The nuclear dynamics in the core-excited state plays a key role in the formation of the spectra. The comparatively large propagation distance in the intermediate core-excited state provides a unique opportunity to observe high-energy vibrational states and hence to probe the full PES. We demonstrate also that frequency detuning, and thereby the control of the scattering time duration and the multimode wave packet propagation offers a possibility to manipulate the dynamics within the selected core-excited PES and that it can be used as an additional tool to probe the ground state PES via various vibrational modes.

Isotope substitution significantly changes the RIXS spectra of the water molecule due to the change in vibrational frequency, followed by distinct nuclear wave packet dynamics. The symmetry breaking in the case of HDO is seen via the breakdown of the selection rules caused by the localization of the vibrations. The replacement of hydrogen by the heavier deuterium affects strongly the ultra-fast nuclear dynamics in the core-excited $4 \mathrm{a}_{1}$ dissociative state and changes the relative intensity and the spectral shape of the so-called "atomic" peak. This dynamical effect plays an important role in understanding the inelastic X-ray scattering of water vapor.

Our work demonstrates that RIXS spectroscopy, taking advantage of the high resolution offered by currently available high performance instrumentation, not only allows us to understand the details and dynamics of the X-ray molecular scattering process as such, but also that it possesses an inherent mode selectivity that can reveal new facts about the vibrational structure and potential energy surfaces of the ground state not reachable by other techniques. Although the system studied here, water, is a simple triatomic system, it is yet sufficiently complex to allow us to generalize these conclusions to common polyatomic gas-phase molecules. The deep insight brought by our findings may also have a bearing on interpreting the properties and structure of other phases, i.e. liquid water and ice.

\section{Author contributions}

V. V. C. and R. C. C. performed all nuclear dynamics simulations and prepared the text of manuscript and figures;
V. V. C. and F. F. G. developed the software and participated in the theoretical analysis and discussion of the results; E. E. and M. O. did all electronic structure calculations; S. E., M. F., M. D., B. K., T. S., A. P. and A. F. suggested and planned the experiment, collected the data and carried out the data analysis; V. K., H. A. and F. G. took the main responsibility for the theoretical modelling and the writing of the paper, in which all authors contributed. All authors reviewed the manuscript.

\section{Acknowledgements}

This work was supported by the Swedish Research Council (VR), The Knut and Alice Wallenberg foundation (Grant No. KAW-2013.0020), and the Carl Tryggers foundation (CTS 15:266). V. V. C. and R. C. C. acknowledge the Conselho Nacional de Desenvolvimento Científico e Tecnológico (CNPq-Brazil); F. G. and V. K. acknowledge support within the State contract of the Russian Federation Ministry of Education and Science for Siberian Federal University for scientific research in 2017-2019 (Project 3.2662.2017); M. D. and T. S. would like to acknowledge the funding from the Swiss National Science Foundation within the D-A-CH programme (SNSF Research Grant 200021L 141325). S. E. and A. F. acknowledge funding from the ERC-ADG-2014-Advanced Investigator Grant-No. 669531 EDAX under the Horizon 2020 EU Framework, Programme for Research and Innovation. M. O. and A. F. acknowledge partial funding by the Helmholtz Virtual Institute VI419 "Dynamic Pathways in Multidimensional Landscapes". The experiments were performed at the ADRESS beam line of the Swiss Light Source at the Paul Scherrer Institute. The calculations were performed on resources provided by the Swedish National Infrastructure for Computing (SNIC).

\section{References}

1 J. Tennyson, N. F. Zobov, R. Williamson, O. L. Polyansky and P. F. Bernath, J. Phys. Chem. Ref. Data, 2001, 30, 735.

2 P. F. Bernath, Phys. Chem. Chem. Phys., 2002, 4, 1501-1509.

3 P. Maksyutenko, M. Grechko, T. R. Rizzo and O. V. Boyarkin, Philos. Trans. R. Soc., A, 2012, 370, 2710-2727.

4 L. Lodi and J. Tennyson, J. Phys. B: At., Mol. Opt. Phys., 2010, 43, 133001.

5 P. Maksyutenko, J. S. Muenter, N. F. Zobov, S. V. Shirin, O. L. Polyansky, T. R. Rizzo and O. V. Boyarkin, J. Chem. Phys., 2007, 126, 10-14.

6 M. Grechko, O. V. Boyarkin, T. R. Rizzo, P. Maksyutenko, N. F. Zobov, S. V. Shirin, L. Lodi, J. Tennyson, A. G. Császár and O. L. Polyansky, J. Chem. Phys., 2009, 131, 221105.

7 J. Nordgren, L. P. Werme, H. Ågren, C. Nordling and K. Siegbahn, J. Phys. B: At. Mol. Phys., 1975, 8, L18-L19.

8 H. Ågren, S. Svensson and U. Wahlgren, Chem. Phys. Lett., 1975, 35, 336-344.

9 J. Nordgren and H. Ågren, Comments At. Mol. Phys., 1984, 14, 203. 
10 G. Fronzoni, M. Stener, A. Lisini and P. Decleva, Chem. Phys., 1996, 210, 447-459.

11 V. Carravetta and H. Ågren, Phys. Rev. A: At., Mol., Opt. Phys., 1987, 35, 1022-1032.

12 A. Cesar, H. Ågren and V. Carravetta, Phys. Rev. A: At., Mol., Opt. Phys., 1989, 40, 187-206.

13 A. Hiraya, K. Nobusada, M. Simon, K. Okada, T. Tokushima, Y. Senba, H. Yoshida, K. Kamimori, H. Okumura, Y. Shimizu, A.-L. Thomas, P. Millie, I. Koyano and K. Ueda, Phys. Rev. A: At., Mol., Opt. Phys., 2001, 63, 042705.

14 I. Hjelte, M. N. Piancastelli, R. F. Fink, O. Björneholm, M. Bässler, R. Feifel, A. Giertz, H. Wang, K. Wiesner, A. Ausmees, C. Miron, S. L. Sorensen and S. Svensson, Chem. Phys. Lett., 2001, 334, 151-158.

15 S. Myneni, Y. Luo, L. Å. Näslund, M. Cavalleri, L. Ojamäe, H. Ogasawara, A. Pelmenschikov, P. Wernet, P. Väterlein, C. Heske, Z. Hussain, L. G. M. Pettersson and A. Nilsson, J. Phys.: Condens. Matter, 2002, 14, L213-L219.

16 L. Inhester, C. F. Burmeister, G. Groenhof and H. Grubmüller, J. Chem. Phys., 2012, 136, 144304.

17 S. Petretti, A. Saenz, A. Castro and P. Decleva, Chem. Phys., 2013, 414, 45-52.

18 F. Gel'mukhanov and H. Ågren, Phys. Rep., 1999, 312, 87-330.

19 L. J. P. Ament, M. van Veenendaal, T. P. Devereaux, J. P. Hill and J. van den Brink, Rev. Mod. Phys, 2011, 83, 705-767.

20 A. Pietzsch, Y.-P. Sun, F. Hennies, Z. Rinkevicius, T. Karlsson, H. O. Schmitt, V. Strocov, J. Andersson, B. Kennedy, J. Schlappa, A. Föhlisch, J.-E. Rubensson and F. Gel'mukhanov, Phys. Rev. Lett., 2011, 106, 153004.

21 M. Le Tacon, A. Bosak, S. M. Souliou, G. Dellea, T. Loew, R. Heid, K.-P. Bohnen, G. Ghiringhelli, M. Krisch and B. Keimer, Nat. Phys., 2014, 10, 52-58.

22 J.-H. Guo, Y. Luo, A. Augustsson, J.-E. Rubensson, C. Såthe, H. Ågren, H. Siegbahn and J. Nordgren, Phys. Rev. Lett., 2002, 89, 137402.

23 S. Kashtanov, A. Augustsson, Y. Luo, J.-H. Guo, C. Såthe, J.-E. Rubensson, H. Siegbahn, J. Nordgren and H. Ågren, Phys. Rev. B: Condens. Matter Mater. Phys., 2004, 69, 24201.

24 T. Tokushima, Y. Harada, O. Takahashi, Y. Senba, H. Ohashi, L. G. M. Pettersson, A. Nilsson and S. Shin, Chem. Phys. Lett., 2008, 460, 387-400.

25 J. Guo and Y. Luo, J. Electron. Spectrosc. Relat. Phenom., 2010, 177, 181-191.

26 A. Pietzsch, F. Hennies, P. S. Miedema, B. Kennedy, J. Schlappa, T. Schmitt, V. N. Strocov and A. Föhlisch, Phys. Rev. Lett., 2015, 114, 088302.

27 M. Odelius, H. Ogasawara, D. Nordlund, O. Fuchs, L. Weinhardt, F. Maier, E. Umbach, C. Heske, Y. Zubavichus, M. Grunze, J. D. Denlinger, L. G. M. Pettersson and A. Nilsson, Phys. Rev. Lett., 2005, 94, 227401.

28 O. Fuchs, M. Zharnikov, L. Weinhardt, M. Blum, M. Weigand, Y. Zubavichus, M. Bär, F. Maier, J. D. Denlinger, C. Heske, M. Grunze and E. Umbach, Phys. Rev. Lett., 2008, 100, 027801.

29 Y. Harada, T. Tokushima, Y. Horikawa, O. Takahashi, H. Niwa, M. Kobayashi, M. Oshima, Y. Senba, H. Ohashi,
K. T. Wikfeldt, A. Nilsson, L. G. M. Pettersson and S. Shin, Phys. Rev. Lett., 2013, 111, 1-5.

30 M. Simon, L. Journel, R. Guillemin, W. C. Stolte, I. Minkov, F. Gel'mukhanov, P. Sałek, H. Ågren, S. Carniato, R. Taieb, a. C. Hudson and D. W. Lindle, Phys. Rev. A: At., Mol., Opt. Phys., 2006, 73, 20706.

31 F. Hennies, A. Pietzsch, M. Berglund, A. Föhlisch, T. Schmitt, V. Strocov, H. O. Karlsson, J. Andersson and J.-E. Rubensson, Phys. Rev. Lett., 2010, 104, 193002.

32 V. Kimberg, A. Lindblad, J. Söderström, O. Travnikova, C. Nicolas, Y. P. Sun, F. Gel'mukhanov, N. Kosugi and C. Miron, Phys. Rev. X, 2013, 3, 011017.

33 C. Miron, C. Nicolas, O. Travnikova, P. Morin, Y. Sun, F. Gel'mukhanov, N. Kosugi and V. Kimberg, Nat. Phys., 2011, 8, 135-138.

34 Q. Miao, O. Travnikova, F. Gel'mukhanov, V. Kimberg, Y.-P. Sun, T. D. Thomas, C. Nicolas, M. Patanen and C. Miron, J. Phys. Chem. Lett., 2015, 6, 1568-1572.

35 X.-J. Liu, Q. Miao, F. Gel'mukhanov, M. Patanen, O. Travnikova, C. Nicolas, H. Ågren, K. Ueda and C. Miron, Nat. Photonics, 2015, 9, 120-125.

36 H. Sann, T. Havermeier, C. Mueller, H. K. Kim, F. Trinter, M. Waitz, J. Voigtsberger, F. Sturm, T. Bauer, R. Wallauer, D. Schneider, M. Weller, C. Goihl, J. Tross, K. Cole, J. Wu, M. S. Schoeffler, H. Schmidt-Boecking, T. Jahnke, M. Simon and R. Doerner, Phys. Rev. Lett., 2016, 117, 243002.

37 V. Kimberg, N. Kosugi and F. Gel'mukhanov, J. Chem. Phys., 2009, 130, 114302.

38 J.-C. Liu, C. Nicolas, Y.-P. Sun, R. Flammini, P. O'Keeffe, L. Avaldi, P. Morin, V. Kimberg, N. Kosugi, F. Gel'mukhanov and C. Miron, J. Phys. Chem. B, 2011, 115, 5103-5112.

39 M. Odelius, Phys. Rev. B: Condens. Matter Mater. Phys., 2009, 79, 144204.

40 R. C. Couto, V. Vaz da Cruz, E. Ertan, S. Eckert, M. Fondell, M. Dantz, B. O'Cinneide, T. Schmitt, A. Pietzsch, F. F. Guimarães, H. Ågren, F. Gel'mukhanov, M. Odelius, V. Kimberg and A. Föhlisch, Nat. Commun., 2017, 8, 14165. 41 N. Ignatova, V. Vaz da Cruz, R. C. Couto, E. Ertan, A. Zimin, F. F. Guimaraes, S. Polyutov, H. Ågren, V. Kimberg, M. Odelius and F. Gel'mukhanov, Sci. Rep., 2017, 7, 43891. 42 P. Morin and I. Nenner, Phys. Rev. Lett., 1986, 56, 1913-1916. 43 F. Gel'mukhanov and H. Ågren, Phys. Rev. A: At., Mol., Opt. Phys., 1996, 54, 379-393.

44 G. Ghiringhelli, A. Piazzalunga, C. Dallera, G. Trezzi, L. Braicovich, T. Schmitt, V. N. Strocov, R. Betemps, L. Patthey, X. Wang and M. Grioni, Rev. Sci. Instrum., 2006, 77, 113108.

45 V. N. Strocov, T. Schmitt, U. Flechsig, T. Schmidt, A. Imhof, Q. Chen, J. Raabe, R. Betemps, D. Zimoch, J. Krempasky, X. Wang, M. Grioni, A. Piazzalunga and L. Patthey, J. Synchrotron Radiat., 2010, 17, 631-643.

46 G. Herzberg, Molecular Spectra and Molecular Structure II: Infrared and Raman Spectra of Polyatomic Molecules, Van Nostrand Reinhold, New York, 1945.

47 B. T. Darling and D. M. Dennison, Phys. Rev., 1940, 57, 128-139. 
48 P. Sałek, A. Baev, F. Gel'mukhanov and H. Ågren, Phys. Chem. Chem. Phys., 2003, 5, 1-11.

49 P. Sałek, Comput. Phys. Commun., 2003, 150, 85-98.

50 F. Gel'mukhanov, P. Sałek, T. Privalov and H. Ågren, Phys. Rev. A: At., Mol., Opt. Phys., 1999, 59, 380-389.

51 V. C. Felicíssimo, F. F. Guimarães, F. Gel'mukhanov, A. Cesar and H. Ågren, J. Chem. Phys., 2005, 122, 094319.

52 P. Jensen, J. Chem. Soc., Faraday Trans. 2, 1988, 84, 1315-1340.

53 H. Ågren, A. Flores-Riveros, H. Jørgen and A. Jensen, Phys. Scr., 1989, 40, 745-750.

54 J. Olsen, P. Jørgensen and J. Simons, Chem. Phys. Lett., 1990, 169, 463-472.

55 P.-Å. Malmqvist, A. Rendell and B. O. Roos, J. Phys. Chem., 1990, 94, 5477-5482.

56 P.-Å. Malmqvist, K. Pierloot, A. R. M. Shahi, C. J. Cramer and L. Gagliardi, J. Chem. Phys., 2008, 128, 204109.

57 P.-Å. Malmqvist, B. O. Roos and B. Schimmelpfennig, Chem. Phys. Lett., 2002, 357, 230-240.

58 P.-Å. Malmqvist and B. O. Roos, Chem. Phys. Lett., 1989, 155, 189-194.

59 B. O. Roos, R. Lindh, P.-Å. Malmqvist, V. Veryazov and P. O. Widmark, J. Phys. Chem. A, 2004, 108, 2851-2858.

60 B. A. Hess, Phys. Rev. A: At., Mol., Opt. Phys., 1986, 33, 3742-3748.

61 M. Douglas and N. M. Kroll, Ann. Phys., 1974, 82, 89-155.

62 B. Schimmelpfenning, AMFI - an atomic mean field integral program, University of Stockholm, Stockholm, Sweden, 1996.

63 M. Rubio, L. Serrano-Andrés and M. Merchán, J. Chem. Phys., 2008, 128, 104305.

64 L. Weinhardt, E. Ertan, M. Iannuzzi, M. Weigand, O. Fuchs, M. Bär, M. Blum, J. D. Denlinger, W. Yang, E. Umbach, M. Odelius and C. Heske, Phys. Chem. Chem. Phys., 2015, 17, 27145-27153.

65 F. Aquilante, J. Autschbach, R. K. Carlson, L. F. Chibotaru, M. G. Delcey, L. De Vico, I. Fdez. Galván, N. Ferré, L. M. Frutos, L. Gagliardi, M. Garavelli, A. Giussani, C. E. Hoyer, G. Li Manni, H. Lischka, D. Ma, P.-Å. Malmqvist, T. Müller, A. Nenov, M. Olivucci, T. B. Pedersen, D. Peng, F. Plasser, B. Pritchard, M. Reiher, I. Rivalta, I. Schapiro, J. Segarra-Mart, M. Stenrup, D. G. Truhlar, L. Ungur, A. Valentini, S. Vancoillie, V. Veryazov, V. P. Vysotskiy, O. Weingart, F. Zapata and R. Lindh, J. Comput. Chem., 2015, 37, 506-541.
66 A. G. Császár, E. Mátyus, T. Szidarovszky, L. Lodi, N. F. Zobov, S. V. Shirin, O. L. Polyansky and J. Tennyson, J. Quant. Spectrosc. Radiat. Transfer, 2010, 111, 1043-1064.

67 M. E. Kellman, Annu. Rev. Phys. Chem., 1995, 46, 395-421.

68 D. X. H. Li and H. Guo, J. Chem. Phys., 2004, 121, 4156.

69 B. Brena, D. Nordlund, M. Odelius, H. Ogasawara, A. Nilsson and L. G. M. Pettersson, Phys. Rev. Lett., 2004, 93, 148302.

70 F. F. Guimarães, V. C. Felicíssimo, V. Kimberg, A. Cesar and F. Gel'mukhanov, eSPec wave packet propagation program, Universidade Federal de Minas Gerais (Brazil) and Royal Institute of Technology (Sweden), 2004.

71 F. F. Guimarães, X-ray Spectroscopy of Molecules Driven by Strong Infrared Fields, PhD thesis, School of Biotecnology, KTH-Stockholm, Sweeden, 2006.

72 D. Neuhauser and M. Baer, J. Chem. Phys., 1989, 91, 4651-4657. 73 Y.-P. Sun, Spontaneous and stimulated X-ray raman scattering, PhD thesis, KTH, Stockholm, Sweden, 2011.

74 Y.-P. Sun, F. Hennies, A. Pietzsch, B. Kennedy, T. Schmitt, V. N. Strocov, J. Andersson, M. Berglund, J.-E. Rubensson, K. Aidas, F. Gel'mukhanov, M. Odelius and A. Föhlisch, Phys. Rev. B: Condens. Matter Mater. Phys., 2011, 84, 132202. 75 J. Stöhr, NEXAFS Spectroscopy, Springer, Berlin, 1992.

76 M. Eroms, O. Vendrell, M. Jungen, H.-D. Meyer and L. S. Cederbaum, J. Chem. Phys., 2009, 130, 154307.

77 S. Schreck, A. Pietzsch, B. Kennedy, C. Såthe, P. S. Miedema, S. Techert, V. N. Strocov, T. Schmitt, F. Hennies, J.-E. Rubensson and A. Föhlisch, Sci. Rep., 2016, 7, 20054.

78 F. Gel'mukhanov, T. Privalov and H. Ågren, Phys. Rev. A: At., Mol., Opt. Phys., 1997, 56, 256-264.

79 S. Sundin, F. Kh. Gel'mukhanov, H. Ågren, S. J. Osborne, A. Kikas, O. Björneholm, A. Ausmees and S. Svensson, Phys. Rev. Lett., 1997, 79, 1451-1454.

80 F. Gel'mukhanov, T. Privalov and H. Ågren, Phys. Rev. A: At., Mol., Opt. Phys., 1997, 56, 256-264.

81 O. L. Polyansky, N. F. Zobov, I. I. Mizus, L. Lodi, S. N. Yurchenko, J. Tennyson, A. G. Császár and O. V. Boyarkin, Philos. Trans. R. Soc., A, 2012, 370, 2728-2748.

82 P. Sałek, V. Carravetta, F. Gel'mukhanov and H. Ågren, J. Chem. Phys., 2002, 116, 629-645.

83 L. Weinhardt, A. Benkert, F. Meyer, M. Blum, R. G. Wilks, W. Yang, M. Bar, F. Reinert and C. Heske, J. Chem. Phys., 2012, 136, 144311. 